\title{
The Four Key Challenges of Advanced Multisensor Navigation and Positioning
}

\author{
Paul D Groves, Lei Wang, Debbie Walter, Henry Martin, Kimon Voutsis, Ziyi Jiang \\ University College London (UCL) \\ London, United Kingdom \\ p.groves@ucl.ac.uk
}

\begin{abstract}
The next generation of navigation and positioning systems must provide greater accuracy and reliability in a range of challenging environments to meet the needs of a variety of mission-critical applications. No single navigation technology is robust enough to meet these requirements on its own, so a multisensor solution is required. Although many new navigation and positioning methods have been developed in recent years, little has been done to bring them together into a robust, reliable, and cost-effective integrated system. To achieve this, four key challenges must be met: complexity, context, ambiguity, and environmental data handling. This paper addresses each of these challenges. It describes the problems, discusses possible approaches, and proposes a program of research and standardization activities to solve them. The discussion is illustrated with results from research into urban GNSS positioning, GNSS shadow matching, environmental feature matching, and context detection.
\end{abstract}

Keywords- Integrated Navigation, Multisensor Navigation, UrbanPositioning

\section{INTRODUCTION}

Before the 1990s, electronic navigation mainly concerned the positioning of large vehicles, such as ships and aircraft, in open environments. Different technologies were applied to air and sea navigation, while land navigation was largely manual. The advent of the Global Positioning System (GPS) provided a single technology that could be used for air, land, and sea navigation, and many other positioning applications, such as surveying. However, it was limited in terms of signal penetration and interference vulnerability. Robustness was improved by integrating global navigation satellite systems (GNSS) with established position-fixing and dead-reckoning technologies [1][2]. However, many capability gaps remained, particularly for indoor and urban navigation, and for applications requiring very high solution availability and reliability.

To bridge the gaps, many new positioning techniques have been investigated over the past fifteen years. Examples include:

Lei Wang is jointly funded by the UCL Engineering Faculty Scholarship Scheme and the Chinese Scholarships Council.

Debbie Walter is jointly funded by the UK's Engineering and Physical Sciences Research Council (EPSRC) and Terrafix Ltd.

Henry Martin is jointly funded by EPSRC and BAE Systems.

Kimon Voutsis is jointly funded by EPSRC and Spirent Communications.

Dr Ziyi Jiang was funded by EPSRC.
- Wi-Fi positioning [3][4][5];

- Ultra-wideband (UWB) positioning [6];

- Positioning using phone signals [7][8][9];

- Positioning using television signals and other signals of opportunity (SOOP) [10][11][12][13][14];

- Bluetooth low energy positioning [15];

- Laser-based position fixing and dead reckoning $[16][17][18][19][20][21]$

- Pedestrian dead reckoning (PDR) using step detection [22][23][24][25];

- Pedestrian map matching [26][27][28][29][30][31];

- Magnetic anomaly matching [32][33][34];

- Activity-based map matching [35][36]; and

- GNSS shadow matching [37][38][39][40][41].

There have also been improvements to existing technologies. The hardware required for visual navigation is now inexpensive and many new position-fixing and deadreckoning algorithms have been developed [42][43][44][45][46][47]. Micro-electro-mechanical systems (MEMS) technology has enabled low-cost (albeit lowperformance) inertial sensors [48], while high-precision inertial sensing has been demonstrated in the laboratory using coldatom technology [49][50], and nuclear magnetic resonance (NMR) gyros offer aviation-grade performance with compact sensors [51]. Legacy radio navigation systems, such as Distance Measuring Equipment (DME) [52][53] and Loran (in Europe and South Korea) [54] are being modernized, and Doppler positioning is being reintroduced using Iridium communication satellites [55]. Finally, GNSS has been enhanced through multiple constellations [1], high-sensitivity receivers and network assistance [56], and augmentation by commercial pseudolite systems [57][58][59]. Current trends in navigation and positioning research are reviewed in [60].

The next generation of navigation and positioning systems must provide greater accuracy and reliability in a range of challenging environments to meet the needs of a variety of mission-critical applications. For example, a universal navigation system might be expected to provide position within

\section{(C) IEEE}

The Four Key Challenges of Advanced Multisensor Navigation and Positioning

Paul D Groves; Lei Wang; Debbie Walter; Henry martin; Kimon Voutsis; Ziyi Jiang.

IEEE/ION Position, Location, and Navigation Symposium (PLANS) 2014, Monterey, CA, 5-8 May 2014.

Digital Object Identifier: 10.1109/PLANS.2014.TBD

http://ieeexplore.ieee.org/Xplore/dynhome.jsp?tag=1 
3 meters at any location with a very high reliability. No single positioning technology is capable of meeting the most demanding application requirements. Radio signals may or may not be subject to obstruction, attenuation, reflection, jamming, and/or interference. Known environmental features, such as signs, buildings, terrain height variation, and magnetic anomalies, may or may not be available for positioning. The system could be stationary, carried by a pedestrian, or on any type of land, sea, or air vehicle. Furthermore, for many applications, the environment and host behavior are subject to change. A multisensor solution is thus required.

Although many new navigation and positioning methods have been developed, little has been done to combine them into a robust, reliable, and cost-effective integrated system. To achieve this, the navigation and positioning community must meet four key challenges. These are as follows:

- Complexity - How to find the necessary expertise to integrate a diverse range of technologies, how to combine technologies from different organizations that wish to protect their intellectual property, how to incorporate new technologies and methods without having to redesign the whole system and how to share development effort over a range of different applications.

- Context - How to ensure that the navigation system configuration is optimized for the operating environment and host vehicle (or pedestrian) behavior when both are subject to change.

- Ambiguity - How to handle multiple hypotheses, including measurements of non-unique environmental features, pattern-matching fixes where the measurements match the database at multiple locations, and uncertain signal properties, such as whether reception is direct or non-lineof-sight (NLOS).

- Environmental Data Handling - How to gather, distribute, and store the information needed to identify signals and environmental features and define their points of origin or spatial variation.

Sections II to V describe each of these challenges in turn. In each case, the problem is explained, one or more solutions are proposed, and the issues that must be resolved in order to implement those solutions are discussed. Section III also presents the results of some preliminary context detection experiments while, in Section IV, the ambiguity problem is illustrated using results from several UCL research projects. Section VI then recommends a program of research and standardization activities to address the four challenges.

\section{COMPLEXITY}

\section{A. The Problem}

Achieving robust positioning in challenging environments potentially requires a large number of subsystems. For example, Fig. 1 shows the possible components of a pedestrian navigation system using sensors found in a typical smartphone. Similarly, Fig. 2 shows possible components of a car navigation system using equipment already common on cars and other suitable low-cost sensors [61]. Comparing these figures, some of the technologies are common to pedestrian and road navigation, whereas others are different.

Any multisensor navigation or positioning system needs integration algorithms to obtain the best overall position solution from the constituent subsystems [1]. These algorithms must not only input and combine measurements from a wide range of subsystems, but also calibrate systematic errors in those subsystems. Designing the integration algorithms therefore requires expertise in all of the subsystems, which can be difficult to establish in a single organization. The more subsystems there are, the more of a problem this is.

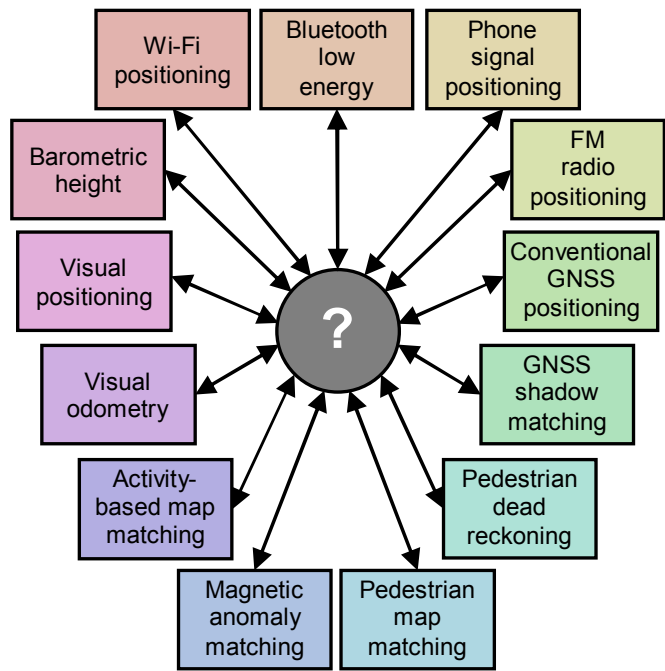

Figure 1. Potential components of a pedestrian navigation system using smartphone sensors

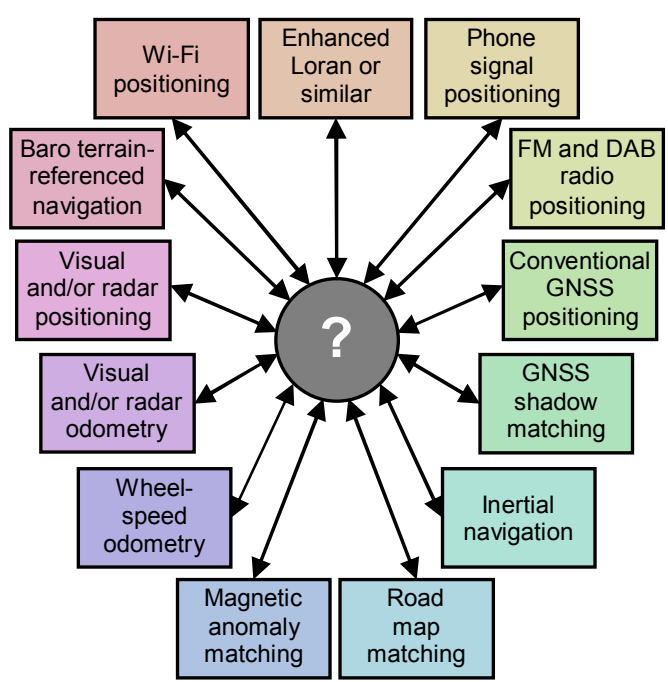

Figure 2. Potential components of a car navigation system using commonly available equipment and other low-cost sensors

The expert knowledge problem is compounded by the fact that different modules in an integrated navigation system are often supplied by different organizations, who may be reluctant to share necessary design information if this is considered to be intellectual property (IP) that must be protected. In a typical smartphone, one company supplies the GNSS chip, another supplies the Wi-Fi positioning service, a third organization 
supplies the mapping, the network operator provides the phone-signal positioning, a fifth company provides the inertial and magnetic sensors and a sixth company produces the operating system. Due to lack of cooperation between these different organizations, useful information gets lost. For example, GNSS pseudo-range measurements are not normally available to "app" developers.

A further issue is reconfigurability. To minimize development costs, manufacturers share algorithms and software across different products, incorporating different subsystems. They also want to minimize the cost of adding new sensors to a product to improve performance. Similarly, researchers want to compare different combinations of subsystems. However, with a conventional system architecture, modifications must be made throughout the integration algorithm each time a subsystem is added, removed, or replaced. The more subsystems there are, the more complex this task becomes.

For a given application, different subsystems may also be used at different times. For example, a smartphone may use Wi-Fi positioning indoors and GNSS outdoors and may deploy different motion constraints and map matching algorithms, depending on whether the device is carried by a pedestrian or traveling in a car. Different integration algorithms for different configurations are more processor efficient, but also require more development effort. Conversely, an all-subsystem integration algorithm is quicker to develop, but can waste processing resources handling inactive subsystems.

\section{B. The Solution: Modular Integration}

The solution to these problems is a modular integration architecture, consisting of a universal integration filter module and a set of configuration modules, one for each subsystem [62]. The integration filter module would be designed by data fusion experts without the need for detailed knowledge of the subsystems. It would accept a number of generic measurement types, such as position fixes and pseudo-ranges, with associated metadata. The configuration modules would be developed by the subsystem suppliers and would convert the subsystem measurements into a format understood by the filter module and supply the metadata. They would also mediate the feedback of information from the integration filter to the subsystems. The metadata comprises the additional information required to integrate the measurements such as

- The measurement type and any coordinate frame(s) used.

- A sensor identification number (to distinguish measurements of the same type from different sensors).

- Statistical properties of the random and systematic measurement errors.

- Identification numbers and locations of transmitters and other landmarks.

A key advantage of this approach is that subsystems may be changed without the need to modify the integration filter. Provided the new subsystem is compatible, all that is needed is the corresponding configuration module.
Fig. 3 shows an example of a modular integration architecture for a combination of conventional GNSS positioning, GNSS shadow matching, Wi-Fi positioning, and PDR. As well as providing measurements and associated statistical data to the integration filter module, the configuration modules feedback relevant information to the subsystems. Shadow matching works by comparing measured and predicted signal availability over a number of candidate positions, so requires a search area to be specified using other positioning technologies [38][39]. PDR uses information from other sensors, where available, to calibrate the coefficients of its step length estimation model and correct for heading drift [24]. Conventional GNSS positioning can also benefit from position and velocity aiding to support acquisition and tracking of weak signals in indoor and urban environments [1][56].

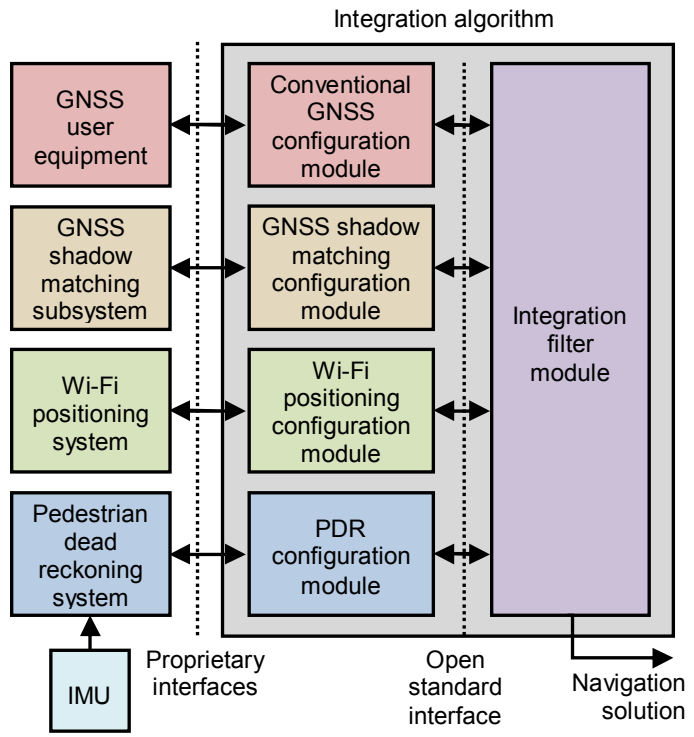

Figure 3. Modular integration of conventional GNSS, shadow matching, PDR, and Wi-Fi positioning for pedestrian navigation (different colors denote potentially different suppliers)

In principle, each subsystem configuration module could simply supply a position fix to the integration filter module with an associated error covariance. However, other forms of measurement generally give better results. For conventional GNSS positioning, the advantages of tightly coupled (rangedomain) integration over loosely coupled (position-domain) are well known [1]. Using pseudo-range measurements from individual satellites in the integration filter minimizes the impact of time-correlated noise and avoids the need for at least 4 satellites to be tracked to provide measurements.

PDR is a dead-reckoning technique, so measures distance traveled rather than position. Consequently, providing measurements of position displacement and direction can avoid cumulative errors in the measurement stream.

GNSS shadow matching and some types of Wi-Fi positioning use the pattern-matching positioning method. This scores an array of candidate position solutions according to the match between the measured and predicted signal availability or signal strength. Although the output of these algorithms is in the position domain, a likelihood distribution can provide more 
information for the integration filter than a simple mean and covariance (see Section IV).

Other navigation and positioning techniques generate further types of measurement, including velocity, attitude, specific force, angular rate, range rate, and bearings and elevations of features. The types of measurement depend on the positioning method, i.e. dead reckoning, proximity, ranging, angular positioning, pattern matching, or Doppler positioning, as detailed in [62].

A universal integration filter must operate without prior knowledge of which measurements it must process and which states it must estimate. Consequently, it must reconfigure its measurement vector, state vector, and associated matrices according to the measurements available, using the metadata supplied by the configuration module. This capability is sometimes called "plug and play" and a number of prototypes have already been developed [63][64][65].

The integration filter must be capable of implementing either error-state or total-state integration, depending on the measurements available [1]. In error-state integration, one of the subsystems, usually a dead-reckoning technology such as inertial navigation, provides a reference navigation solution and the integration filter estimates corrections to that solution using the measurements from other subsystems. In total-state integration, the integration filter estimates the position, velocity, and attitude (where appropriate) directly. In the totalstate case, an additional configuration module is required to provide the universal filter with information on the host vehicle (or pedestrian) dynamic motion.

Modular integration algorithms could form part of a wider modular integrated navigation concept in which subsystem hardware and software is also shared across a wide range of applications. Software sharing could span the consumer, professional, research, and military user communities, though opportunities to share hardware designs are more limited due to the differences in user requirements [62].

\section{Issues to Resolve}

A critical requirement for the successful implementation of modular integration is an open-standard interface for communication between the universal filter and configuration modules. This enables modules produced by different organizations to work together. To realize the full benefits of modular integration, in terms of interoperability and software re-use, there should be a single standard covering the consumer, professional, research, and military user communities and spanning all of the application domains air, sea, land, indoor, underwater, and so forth. A standard developed by one group in isolation is unlikely to meet the needs of the whole navigation and positioning community, while the development of multiple competing standards defeats the main purpose of modular integration.

This interface should be defined in terms of fundamental measurement types, such as position, velocity and range. However, there are many different ways of expressing these. Position may be curvilinear or Cartesian. Cartesian position may be Earth-centered inertial (ECI), Earth-centered Earth- fixed (ECEF), or local. A local coordinate frame may or may not be aligned with north, east, and down. A position fix may also have 1, 2, or 3 dimensions, while $1 \mathrm{D}$ and $2 \mathrm{D}$ fixes are not necessary aligned with the axes of the coordinate frames.

Depending on how the relevant subsystem works, measurements of velocity, position displacement, acceleration, specific force, angular rate, and the bearings and elevations of features may be resolved about the axes of the either the host vehicle or sensor body frame or an external reference frame. Similarly, ranging measurements may be true ranges or pseudo-ranges. Furthermore ranging and angular positioning measurements may be differenced across transmitters or landmarks, differenced across receivers or sensors, or double differenced across both.

A universal interface must support every measurement type that requires different processing by the filter module. However, it need not support formats that are easily convertible. Thus, there is no need to support both the north, east, down and east, north, up conventions. There are two main approaches to defining the fundamental measurement types:

- A minimal number of very generic measurement types with metadata used to describe how these should be processed by the integration filter.

- A large number of more specific measurement types for which the processing methodology is already known.

For example, in the generic approach, there would be only one type of position measurement, but it would have a variable number of components and each axis would be specified in the metadata. Conversely, for the specific approach, 3D position, horizontal position, height, and line fixes would be separate measurement types. A hierarchy of types and sub-types could also be adopted.

For each measurement type, an error specification must be defined. For error sources assumed to be white, a standard deviation or power spectral density (PSD) is required. For correlated errors, such as biases, scale factor and crosscoupling errors, and higher-order systematic errors, information on the time correlation is required alongside variances and covariance information. The interface standard should include every conceivable error source. Error sources that are insignificant for a particular implementation should simply be zeroed by the relevant configuration module. The filter module should then use the error specification to determine which error sources to model and how.

Obtaining reliable navigation sensor error specifications can be difficult. Manufacturers often provide only limited information, while performance in the field can be different from that in the laboratory due to vibration and electromagnetic interference. For new positioning techniques, the error behavior may not be fully understood, while complex error behavior can be difficult to measure. Adaptive estimation techniques [66][67][68] provide only a partial solution. Even where the error behavior is well known, it can too complex to practically model within the estimation algorithm. Arguably, this represents a fifth challenge to the community [69]. 
For subsystems used as the reference in an error-state integration filter, such as an inertial navigation system (INS), the errors will typically be correlated across the different components of the subsystem navigation solution, e.g. position, velocity, and attitude. Furthermore, to represent the error behavior within an integration algorithm, it is necessary to model the error properties of the underlying sensors, accelerometers and gyroscopes in the case of inertial navigation [1]. Thus, it is likely that additional compound measurement types for reference system data will be needed.

For pseudo-range measurements, an issue to consider is the synchronization of different transmitter and receiver clocks. Clocks in receivers for different types of signal, such as GNSS and Loran, may or may not be synchronized with each other. Also, the transmitter clocks are typically synchronized in groups. For example, the GPS satellite clocks are synchronized with each other, as are the GLONASS satellite clocks, but GLONASS is not currently synchronized with GPS. For optimal integration of pseudo-ranges from different sources, this information must be conveyed to the integration filter.

The interface standard for communication between the filter and configuration modules must also support feedback of information from the integration filter to the subsystems, via the configuration modules. The integrated position, velocity, and attitude solution, with its associated error covariance, is useful for aiding many different subsystems. Therefore, a generic standard for this should be defined. Conversely, the feedback to the subsystems of calibration parameters estimated by the integration algorithm is sensor specific, so should be incorporated in the definitions of the fundamental measurement types. Note that closed-loop correction of subsystem errors is often essential to maintain the validity of linearization approximations within the integration filter [1].

The user requirements, such as accuracy, integrity, continuity, solution availability, update rate, and power consumption, can vary greatly between applications. For example, accuracy is important for surveying, integrity for civil aviation, solution availability for many military applications, and power consumption for many consumer applications. This impacts the design of the whole navigation system: the integration filter, the configuration modules, and the subsystems. Different modules could be used for different applications. However, to reap the full benefits of a modular approach, the components should be able to adapt to different user requirements. This is particularly important for devices, such as smartphones, which must switch between the requirements of different applications. Fig. 4 shows how requirements information can be disseminated in a modular integrated navigation system. The adaptation of GNSS user equipment to varying user requirements is discussed in [70].

An open-standard interface specification should be able to handle measurements from and feedback to any conceivable navigation and positioning system. However, it is not practical to expect every filter module to handle all measurement types because of the development effort required. Similarly, there will be differences in the sizes of errors that an integration filter can handle and in its capability to handle non-Gaussian error distributions. For example, an integration algorithm based on a

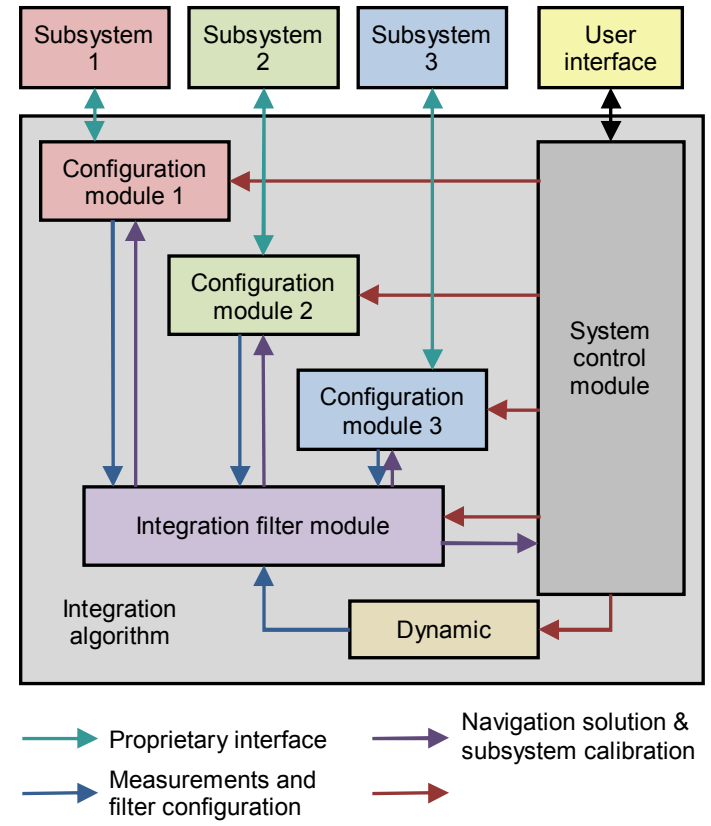

Figure 4. Modular integration architecture incorporating requirements information distribution

particle filter has different capabilities from one based on an extended Kalman filter. Variations in fault detection and integrity monitoring capability can also be expected. Consequently, there must be a capability specification for each filter module and a protocol for handling mismatches between the measurements and the filter module. For many applications, there will also be a need to certify the filter module to ensure it actually has the capabilities claimed for it.

\section{CONTEXT}

\section{A. The Problem}

Context is the environment that a navigation system operates in and the behavior of its host vehicle or user. Examples include a pedestrian walking (behavior) in an urban street (environment), a car driving at highway speeds (behavior) on an open road (environment), and an airliner flying (behavior) high above an ocean (environment).

Context is critical to the operation of a navigation or positioning system. The environment affects the types of signals available. For example, GNSS reception is poor indoors while $\mathrm{Wi}-\mathrm{Fi}$ is not widely available outside towns and cities. In underwater environments, most radio signals cannot propagate so acoustic signals are used instead. Processing techniques can also be context dependent. For example, in open environments, non-line-of-sight (NLOS) reception of GNSS signals or multipath interference may be detected using consistency checking techniques based on sequential elimination. However, in dense urban areas, more sophisticated algorithms are required [71] and may be enhanced using 3D city models [72][73][74][75][76]. Similarly, GNSS shadow matching only works in outdoor urban environments [38].

Navigation using environmental feature matching is inherently context-dependent as different types of feature are 
available in different environments. Suitable algorithms, databases, and sensors must be selected. For example, terrain referenced navigation (TRN) uses radar or laser scanning in the air [17][77][78], sonar or echo sounding at sea [79], and barometric pressure on land [61][80]. Map matching requires different approaches for cars [81], trains [82], and pedestrians [26][27]. Similarly, algorithms and databases for image-based navigation depend on the types of feature available, which vary with the environment [42][43][44][45][46][47].

Behavioral context is also important and can contribute additional information to the navigation solution. For example, cars normally remain on the road, effectively removing one dimension from the position solution. Their wheels also impose constraints on the way they can move, reducing the number of inertial sensors required to measure their motion [83][84]. Similarly, PDR using step detection depends inherently on the characteristics of human walking [23][24]. Trying to use PDR for vehicle navigation or vehicle motion constraints for pedestrian navigation will result in errors.

Host vehicle behavior is also important for tuning the dynamic model within a total-state navigation filter and for detecting faults through discrepancies between measured and expected behavior [1]. Within a GNSS receiver, the behavior can be used to set tracking loop bandwidths and coherent correlator accumulation intervals, and to predict the temporal variation of multipath errors [85]. The antenna placement on a vehicle or person [86] can also affect performance.

Historically, context was implicit; a navigation system was designed to be used in a particular type of vehicle, handling its associated behavior and environments. However, many navigation systems now need to operate in a variety of different contexts. For example, a smartphone moves between indoor and outdoor environments and can be stationary, on a pedestrian, or in a vehicle. Similarly a small surveillance drone may operate from above, amongst buildings, or even indoors. Furthermore, as discussed in Section II, there is a growing need to re-use hardware and software modules across multiple applications to reduce development and production costs. At the same time, most of the new positioning techniques developed to enable navigation in challenging environments (see Section I), are context-dependent. To make use of these techniques in practical applications (as opposed to research demonstrators), it is necessary to know the context.

\section{B. The Solution: Context-Adaptive Navigation}

The solution to the problem of using context-dependent navigation techniques in variable-context applications is context-adaptive navigation [1][87]. As shown in Fig. 5, the navigation system detects the current environmental and behavioral context and, in real time, reconfigures its algorithms accordingly. For example, different radio positioning signals and techniques may be selected, inertial sensor data may be processed in different ways, different map-matching algorithms may be selected, and the tuning of the integration algorithms may be varied.

Previous work on context-adaptive navigation and positioning has focused on individual subsystems and concerned either behavioral or environmental context, not both.

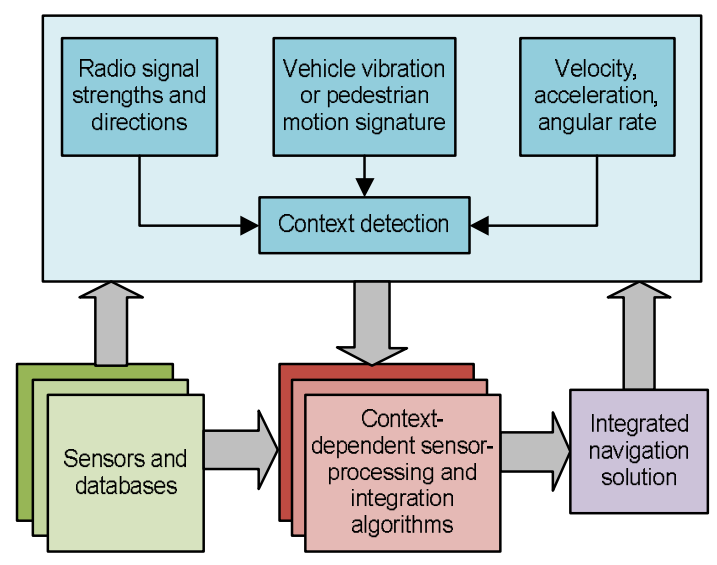

Figure 5. A context-adaptive navigation system [1]

For example, there has been substantial research into classifying pedestrian motion using inertial sensors to enable PDR algorithms using step detection to estimate the distance travelled from the detected motion [88][89][90][91]. The context information may also be used for non-navigation purposes [92][93].

Typically, orientation-independent signals are generated from the accelerometer and gyro outputs. Statistics such as the mean, standard deviation, root mean squared (RMS), interquartile range, mean absolute deviation, maximum-minimum, maximum magnitude, number of zero crossings, and number of mean crossings are then determined from a few seconds of data. Frequency-domain statistics may also be used. Finally, a pattern recognition algorithm is used to match these parameters to the stored characteristics of different combinations of activity types and sensor locations.

Detection of road-induced vibration using accelerometers has been used to determine whether or not a land vehicle is stationary [94][95], while a calibrated yaw-axis gyro can be used to determine when a vehicle is travelling in a straight line [96]. Recent work at UCL has also shown that vibration spectra derived from accelerometer measurements can be used to distinguish when a device is on a table, held by a stationary pedestrian, and placed in a stationary car or bus [87].

Indoor and outdoor environments may be distinguished using GNSS carrier-power-to-noise-density ratio $\left(\mathrm{C} / \mathrm{N}_{0}\right)$ measurements [85] or a Rician K-Factor estimator [97]. Recent work at UCL has confirmed this over a wider range of environments and shown that the GNSS $C / N_{0}$ measurements can also be used to distinguish different types of outdoor environments, such as urban and open [87].

Wi-Fi signals might also be used for environmental context detection. Early experiments suggested that indoor and outdoor environments might be distinguished using a combination of the number of access points received, the average signal-tonoise ratio (SNR), and the SNR standard deviation [98]. By contrast, in recent UCL experiments across a wider range of environments, it was difficult to identify differences between the indoor and outdoor datasets [87]. However, differences between types of outdoor environment were observed and it was found that the types of access points received could be used to distinguish between residential and business districts. 
Further context detection experiments are described in Section III.C. Despite the work done with individual sensors, a multisensor integrated navigation system that adapts to both environmental and behavioral context remains at the concept stage. Realizing this in a practical system requires both effective context determination and a standard set of context categories. These issues are explored in Section III.D, while Section 0 discusses how to incorporate context adaptivity in a modular integration architecture.

\section{Context Detection Experiments}

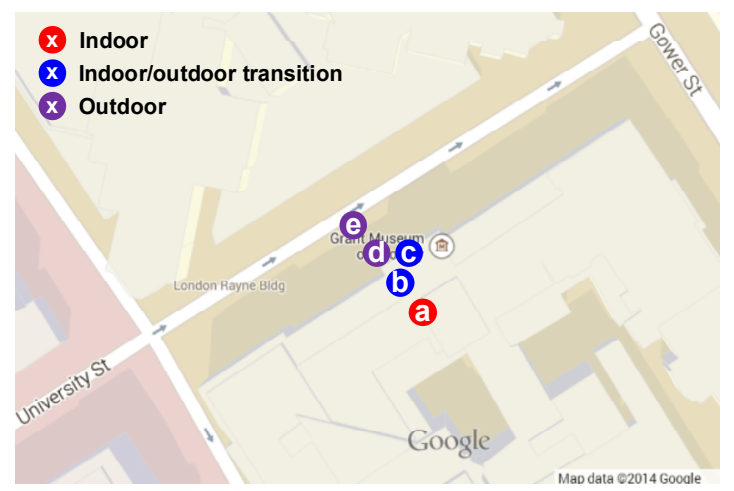

Figure 6. Locations for the GNSS indoor/outdoor context detection experiment at UCL's Grant Museum of Zoology
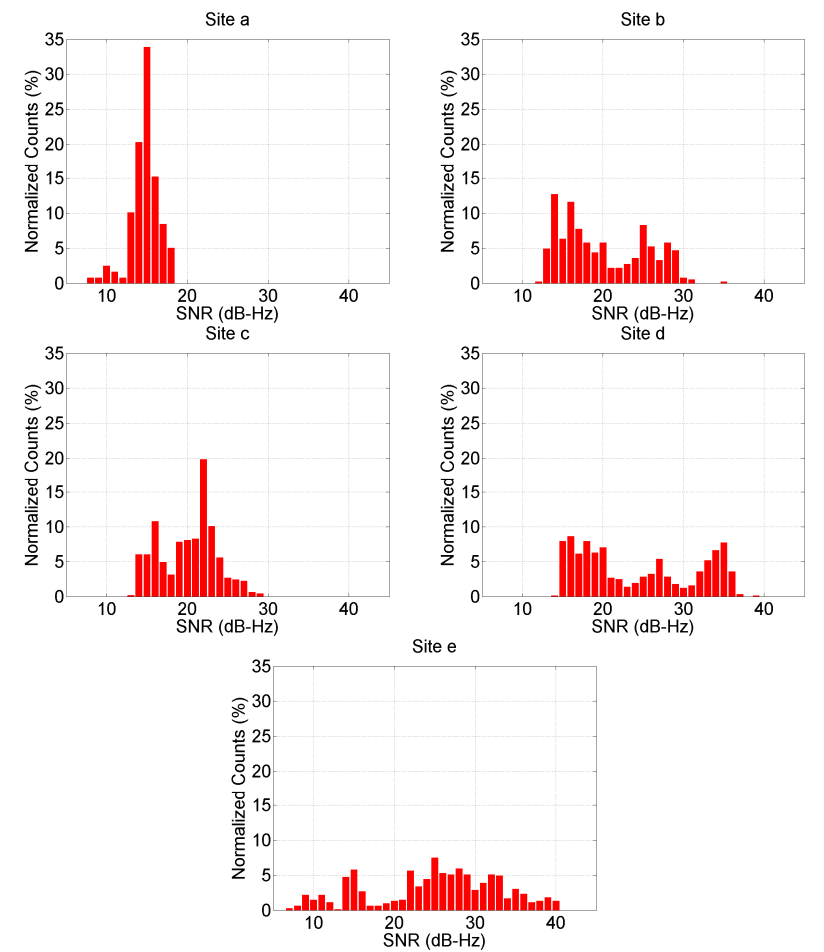

Figure 7. GNSS $C / N_{0}$ measurement distributions at sites inside and immediately outside UCL's Grant Museum of Zoology

Experiments have been conducted to assess the feasibility of using GNSS and Wi-Fi for distinguishing indoor and outdoor environments and accelerometers for distinguishing a number of behavioral contexts. These directly follow the experiments described in [87].

\section{1) Indoor/Outdoor Context Detection using GNSS}

GNSS data was collected at five locations inside and immediately outside UCL's Grant Museum of Zoology; these are shown in Fig. 6, $C / N_{0}$ measurement data was collected from all GPS and GLONASS signals received by a Samsung Galaxy S3 Android smartphone. About 60s of data was collected at each site. Fig. 7 presents histograms of the $C / N_{0}$ measurements and Table I lists the means and standard deviations.

TABLE I. MEANS AND STANDARD DEVIATIONS OF GNSS $C / N_{0}$ MEASUREMENTS INSIDE AND OUTSIDE UCL'S GRANT MUSEUM OF ZOOLOGY

\begin{tabular}{|l|c|c|}
\hline \multicolumn{1}{|c|}{ Site } & Mean $\boldsymbol{C} / \boldsymbol{N}_{\boldsymbol{0}}$ & $\boldsymbol{C} / \boldsymbol{N}_{\boldsymbol{0}} \boldsymbol{S D}$ \\
\hline a (Deep indoors) & $14.7 \mathrm{~dB}-\mathrm{Hz}$ & $1.8 \mathrm{~dB}-\mathrm{Hz}$ \\
\hline b (Inside, near entrance) & $20.0 \mathrm{~dB}-\mathrm{Hz}$ & $5.3 \mathrm{~dB}-\mathrm{Hz}$ \\
\hline c (Inside, in the doorway) & $20.1 \mathrm{~dB}-\mathrm{Hz}$ & $3.5 \mathrm{~dB}-\mathrm{Hz}$ \\
\hline d (Outside, on entrance steps) & $24.4 \mathrm{~dB}-\mathrm{Hz}$ & $7.3 \mathrm{~dB}-\mathrm{Hz}$ \\
\hline e (Outside, by the kerb) & $25.0 \mathrm{~dB}-\mathrm{Hz}$ & $7.9 \mathrm{~dB}-\mathrm{Hz}$ \\
\hline
\end{tabular}

As expected, the average received $C / N_{0}$ is lower indoors than outdoors and lower deep indoors than near the entrance. Furthermore, the standard deviation of the $C / N_{0}$ measurements is larger outdoors than indoors and also larger near the entrance to the building than deep indoors. Both trends are consistent with previous results collected in a range of different environments [87]. Thus, both the mean and the standard deviation of the measured $C / N_{0}$ across all GNSS satellites tracked are useful both for detecting indoor and outdoor contexts and for distinguishing between different types of indoor environment. Context detection should be more reliable if both the mean and standard deviation are used.

\section{2) Indoor/Outdoor Context Detection using Wi-Fi}

Tests in and around several UCL buildings have shown no clear relationship between Wi-Fi SNRs and environmental context, confirming previous results [87]. However, as the environment changes, there is a rapid change in the Wi-Fi SNRs over a few epochs. For a user moving from inside to outside of a particular building, those signals which originate inside go from strong to weak, while many of those from neighboring buildings become stronger. Consequently, Wi-Fi signals could potentially be used to detect context changes instead of the absolute context. Thus is useful for improving the overall robustness of context determination.

To test this, Wi-Fi SNR data was collected on a Samsung Galaxy S3 smartphone, along a route with both indoor and outdoor sections. One step was taken at each epoch. The magnitude of the SNR differences over intervals of one to six epochs was computed for each received signal. An overall "context change" score was then calculated at each epoch, comprising the weighted mean across all signals and innovations. Shorter durations were given greater weighting.

The "context change" score results are presented in Fig. 8. The large blue blocks indicate when the user was outside and the smaller blue block shows when the user was in the building's basement, a very different Wi-Fi environment. As can be seen, there are clear peaks in the "context change" score whenever the user moves between indoor and outdoor contexts. 
However, there are also peaks when the user enters and leaves the basement, so the technique is sensitive to false positives and must be combined with other context detection techniques to be used reliably.

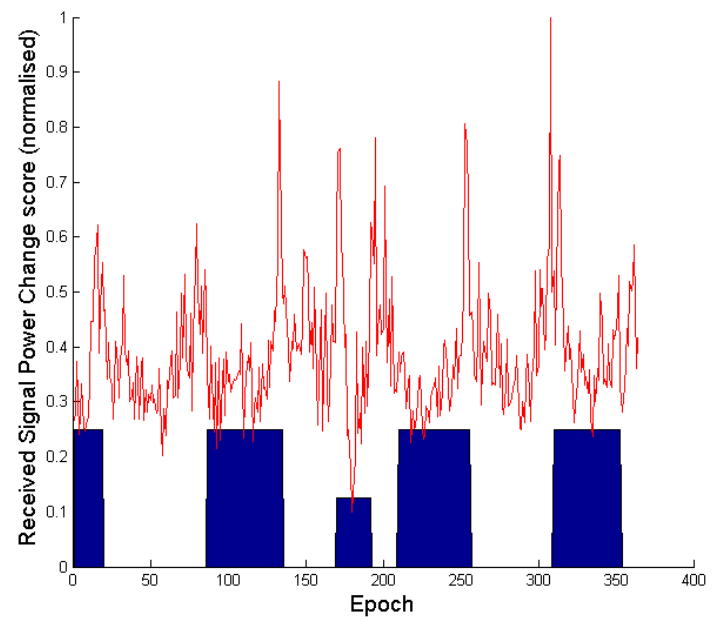

Figure 8. "Context change" score computer from Wi-Fi SNR measurements

\section{3) Behavioral context detection using accelerometers}

In [87], it was shown that accelerometer vibration spectra can be used to distinguish when a stationary device is in a road vehicle, held by a pedestrian, or on a table, and also to determine when the car is moving. Here, further results from a train, a car, an escalator and an elevator are presented.

Specific force data was collected using an Xsens MTi-G IMU/GNSS device. However, any accelerometers, including smartphone sensors, are potentially suitable. For the escalator and elevator experiments, the magnitude of the specific force was analyzed while, for the train and car experiments, the individual components were examined. In all cases, the mean of the specific force measurements was subtracted to remove most of the gravity, which dominates the measurements, giving specific force residuals, from which vibration is easy to identify. A discrete Fourier transform was then applied using the MATLAB function $\mathrm{fft}$. Note that this integrates the specific force residuals.

Fig. 9 and Fig. 10 respectively show the vibration spectra of a stationary Vauxhall Insignia car, and a stationary urban electric train. In each case, the $\mathrm{x}$-axis was pointing forward, the $\mathrm{y}$-axis to the right and the z-axis down. The car exhibits a lot of vibration at frequencies above $10 \mathrm{~Hz}$ due to its engine, whereas the dominant train vibration peak is around $1.5 \mathrm{~Hz}$, with smaller peaks at $15 \mathrm{~Hz}, 25 \mathrm{~Hz}, 33 \mathrm{~Hz}$, and $50 \mathrm{~Hz}$, the mains power frequency. Thus, the two vehicles are very different.

Fig. 11 shows the vibration spectrum of the car moving on a high-speed road, while Fig. 12 shows the vibration spectrum of the train travelling between Euston and Watford Junction stations in the London area. As might be expected, both show much more vibration when moving than when stationary. For the car, there are broad peaks below $15 \mathrm{~Hz}$ due to road vibration and above $15 \mathrm{~Hz}$ due to engine vibration. For the train, vibration below $4 \mathrm{~Hz}$ dominates. Thus, there is clear scope to distinguish between the two types of vehicle.

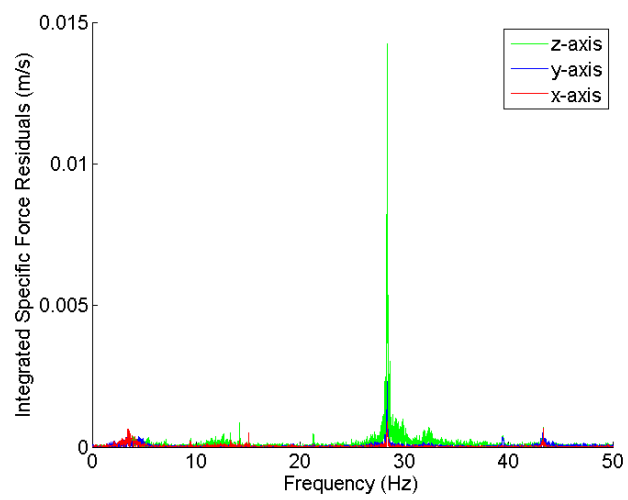

Figure 9. Specific force frequency spectrum of a stationary car

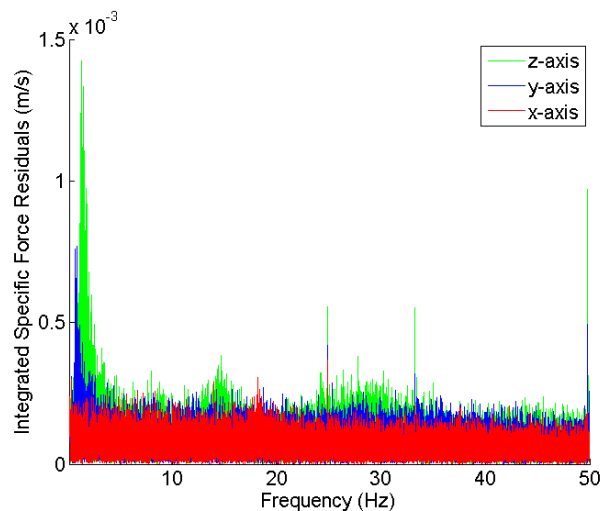

Figure 10. Specific force frequency spectrum of a stationary train

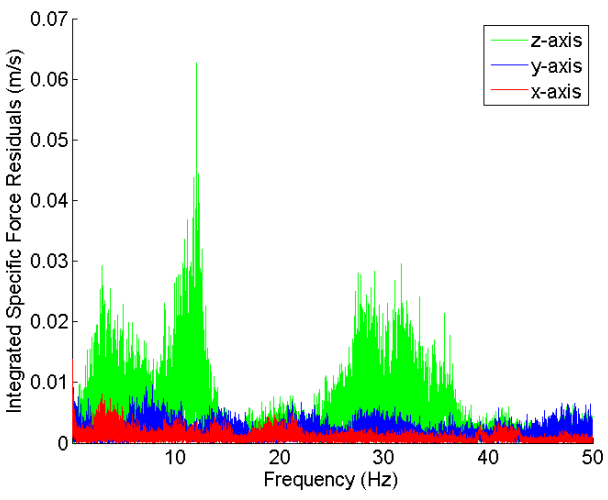

Figure 11. Specific force frequency spectrum of a car traveling on a highspeed road

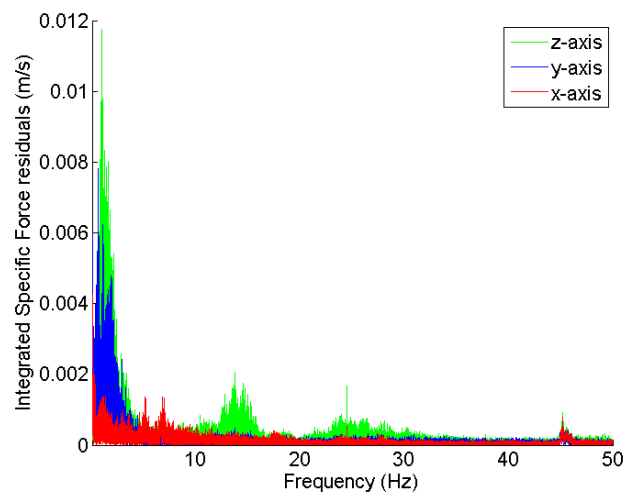

Figure 12. Specific force frequency spectrum of a moving train 
Fig. 13 and Fig. 14 show the vibration spectra in a moving elevator at Hampstead London Underground station and on an escalator at Angel station, respectively. In both cases, the IMU was in the trouser pocket of a pedestrian. Contrary to reports in [16], these are very different. The elevator spectrum is dominated by low-frequency acceleration. Distinct periods of acceleration and deceleration can be observed in the time domain By contrast, the escalator motion is subject to vibration at a range of frequencies below $30 \mathrm{~Hz}$. The resonant frequencies vary between individual escalators.

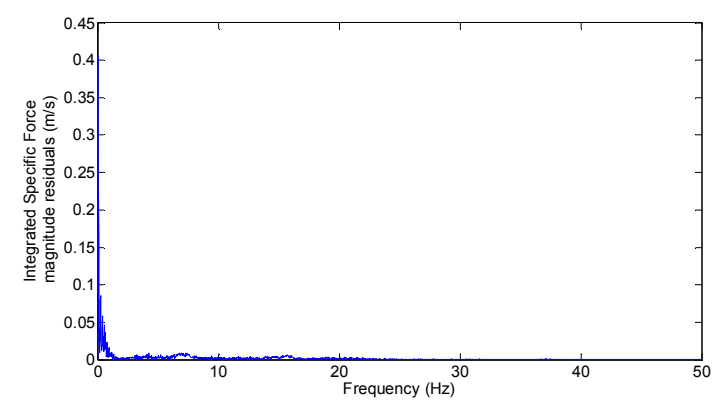

Figure 13. Specific force frequency spectrum in a moving elevator

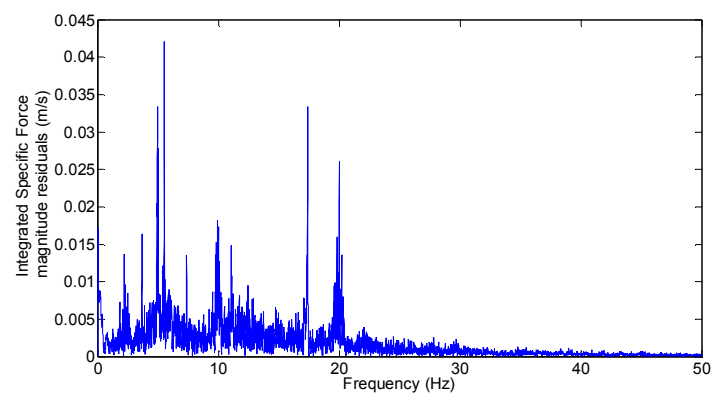

Figure 14. Specific force frequency spectrum on an escalator

\section{Issues to Resolve}

\section{1) Context Categorization}

To implement a multisensor navigation system with many different subsystems adapting to the context and contributing to the context determination process, a common set of context categories and their definitions must be established. As different modules will often be produced by different organizations, standardization across whole the navigation and positioning community is required. Standard context definitions are also needed to enable software modules to be reused across multiple applications.

The first step in the standardization process is to establish a framework suitable for navigation and positioning. Each context category must map to a configuration of the navigation system; otherwise, it serves no purpose. Multiple categories may map to the same configuration as different navigation systems will respond to different context information. In an autonomous context-adaptive navigation system, the context categories must also be distinguishable from each other.

In [87], a five-attribute framework, comprising environment class, environment type, behavior class, vehicle type, and activity type, was proposed. Fig. 15 shows the relationship between the attributes. The environmental and behavioral contexts are treated separately because they perform fundamentally different roles in navigation. Environmental context concerns the availability of signals and other features that may be used for determining position whereas behavioral context is concerned with motion.

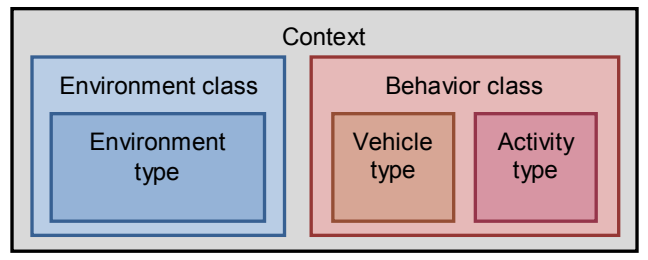

Figure 15. Proposed attributes of a context category [87]

Context may be considered at different levels. Sometimes it is sufficient to consider broad classes such as indoor or aircraft. In other cases, more detail is needed, specifying the type of indoor environment or the type of aircraft. Therefore, a twolevel categorization framework, comprising class and type, was proposed. The behavioral context comprises the vehicle type and the activity undertaken by that vehicle. A common set of classes containing separate vehicle and activity types was thus proposed. For pedestrian navigation, different parts of the body move quite differently, so the sensor location on the body is analogous to the vehicle type.

The broad classes of environmental and behavioral context are relatively obvious. It is therefore proposed that the community adopts the classes listed in Table II. Standardization at the type level requires further research to determine:

- Which context categories a navigation system needs to distinguish between in order to optimally configure itself, and

- Which context categories may be distinguished reliably by context detection and determination algorithms.

Some possible environment, vehicle, and activity types are proposed in [87].

TABLE II. PROPOSED ENVIRONMENT AND BEHAVIOR ClASSES

\begin{tabular}{|c|c|}
\hline Environment Classes & Behavior Classes \\
\hline Indoor & Land Vehicle \\
Land Outdoor & Boat or Ship \\
On Water & Underwater Vehicle \\
Underwater & Aircraft \\
Air & Spacecraft \\
Space & Pedestrian \\
\hline
\end{tabular}

\section{2) Effective Context Determination}

The reliability of current context detection techniques is typically 90-99\%, with some context categories easier to detect than others. For the purposes of controlling a navigation system, this is relatively poor. Furthermore, context detection research projects have typically considered a much smaller range of context categories than a practical context-adaptive navigation system would need. Generally, the more categories there are, the harder it is to distinguish between them. 
To make context determination reliable enough for contextadaptive navigation to be practical, a new approach is needed [87]. Firstly, the context should be detected using as much information as possible, maximizing both the range of sensors used and the number of parameters derived from each sensor.

Environmental context detection experiments have largely focused on GNSS and Wi-Fi signals. Other types of radio signal; environmental features detected using cameras, laser scanners, radar, or sonar; ambient light; sounds; odors; magnetic anomalies, and air pressure could all be used. Context may also be inferred simply by comparing the position solution with a map, provided both are sufficiently accurate [99].

Behavioral context detection experiments have generally used inertial sensors. As shown in Section III, this could be taken further by analyzing different frequency bands and, where possible, separating the forward, transverse, and vertical components. Other motion sensing techniques, such as visual odometry and wheel-speed odometry could be used. Context information, such as vehicle type, can also be determined from the velocity, attitude, and acceleration solutions.

A further line of research is the development of algorithms to detect changes in context as well as absolute context. This is applicable to both the environment and the behavior.

Considering every combination of environment type, vehicle type (or pedestrian sensor location), and activity type, there are potentially tens of thousands of different context categories. This is too many to practically distinguish using context detection techniques alone. However, the number of context categories that must be considered may be reduced substantially by using association, scope, and connectivity information, making the context determination process much more reliable [87].

Association is the connection between the different attributes of context. Certain activities are associated with certain vehicle types and certain behaviors are associated with certain environments. For example, an airliner flies, while a train does not and flying takes place in the air, not at the bottom of the sea. The behavior of a vehicle can also vary with the environment. For example, a car typically travels more slowly, stops more, and turns more in cities than on the highway. Thus, combinations of environment type, vehicle type, and activity type that are not associated in practice, may be eliminated, while weakly associated combinations may be downweighted in the context determination process.

For a particular application, the scope defines each context category to be required, unsupported, or forbidden. The required categories are those that the navigation system must detect and respond to. Unsupported context categories are those that could occur, but need not be detected and responded to. Finally, the forbidden context categories are those that cannot occur. For example, a navigation system permanently fitted to a car cannot be flying or running. Thus, scope definition enables forbidden context categories to be eliminated from the context determination process and required categories to be treated as more likely than unsupported categories.

Connectivity describes the relationship between context categories. If a direct transition between two categories can occur, they are connected. Otherwise, they are not. Thus, stationary vehicle behavior is connected to pedestrian behavior, whereas moving vehicle behavior is not because a vehicle must normally stop to enable a person to get in or out. Context connectivity is directly analogous to the road link connectivity used in map matching [100] and a similar mathematical formulation may be used. In practice, it is best to represent the connectivity as continuously valued transition probabilities rather than in Boolean terms. This facilitates recovery from incorrect context determination and enables rare transitions between context categories to be represented.

Location-dependent connectivity takes the concept a stage further by considering that many transitions between context categories happen at specific places. For example, people normally board and leave trains at stations and fixed-wing aircraft typically require an airstrip to take off and land. Thus context transition probabilities may be modeled as functions of the position solution, provided the positioning and mapping error distributions are adequately modeled and the probability of transitions occurring at unusual locations is considered.

Further examples of context association, scope, and connectivity may be found in [87].

Finally, for maximum robustness, the whole context determination process should be probabilistic, not discrete. The system should maintain a list of possible context category hypotheses, each with an associated probability. Multiple context detection algorithms should be used, each based on different sensor information. The detection algorithms should also output multiple context category hypotheses with associated probabilities. The context determination algorithm should then produce a new list of context category hypotheses and their probabilities by combining:

- The previous list of hypotheses and their probabilities;

- The hypotheses and probabilities output by the context detection algorithms;

- Context association, scope, and connectivity information.

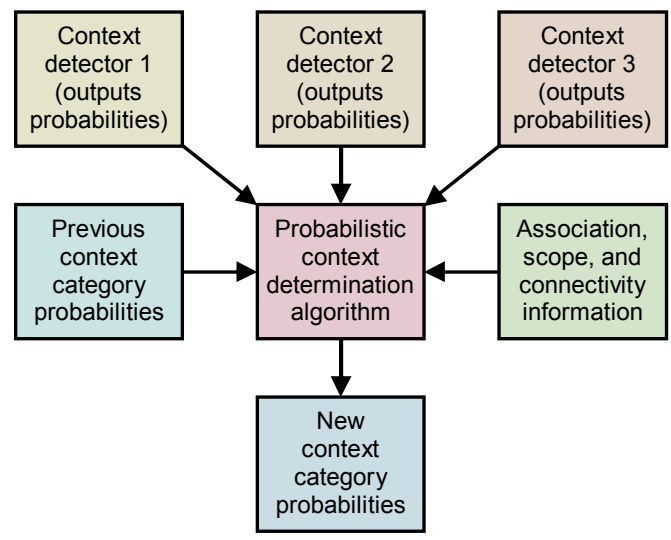

Figure 16. Probabilistic context determination.

A suitable algorithm for this is described in [87], while Fig. 16 illustrates the concept. When there is insufficient information to determine a clear context category, the list of context hypotheses and their probabilities will be output to the 
navigation algorithms. The handling of ambiguous information in navigation systems is the subject of Section IV.

\section{E. Context Adaptivity and Modular Integration}

The practical implementation of a complex multisensor navigation system for a multi-context application requires context-adaptive navigation to be incorporated into a modular multisensor integration architecture (Section II.B). To enable different modules to adapt to changes in context, the architecture shown in Fig. 4 should be extended to supply context information to the configuration modules, integration filter, and dynamic model from the system control module, alongside the user requirements. The configuration modules can then pass the context information onto the subsystems where necessary. The standardization of context categories and their definitions across the navigation and positioning community is essential for this. The distribution of context information is useful even for single-context applications as it enables suppliers to provide modules that are optimized for multiple contexts.

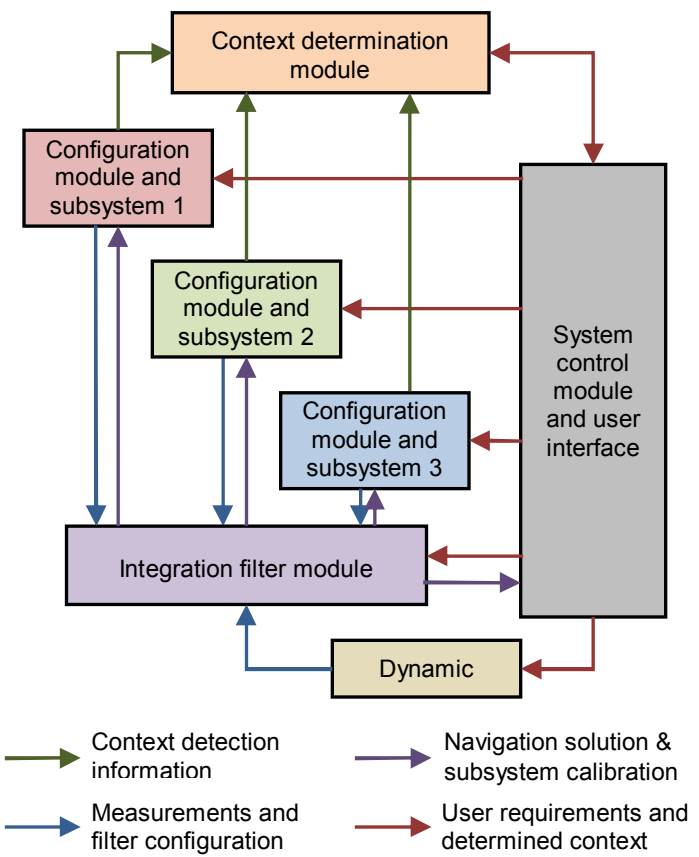

Figure 17. Context-adaptive modular multisensor integration architecture

The modular integration architecture must also support the context detection and determination process, allowing all subsystems to contribute. The configuration modules should therefore provide context detection information to a context determination module, as shown in Fig. 17. The scope information should be supplied by the system control module.

There are three main ways in which context detection could be implemented:

\section{1) Subsystem-based approach}

The context detection algorithms are implemented inside the subsystems or configuration modules with a list of possible context categories and their probabilities sent to the context determination module. This has the advantage of keeping the context determination model generic. However, the subsystem suppliers may consider the need to implement context detection software to be too much of a burden, particularly if they don't consider context adaptivity to be a core requirement.

\section{2) Context-determination-module-based approach}

The context detection algorithms are implemented in the context-determination module with the subsystems providing the necessary data. This is straightforward for subsystems such as an IMU or a GNSS receiver where the necessary information is commonly output. However, for some of the newer navigation and positioning technologies, the subsystem suppliers may consider the underlying data to be proprietary. For other subsystems, such as image-based navigation, the volume of data could be a problem. An advantage of this approach is that context detection algorithms may use data from more than one navigation subsystem. A variation on this architecture would comprise separate modules for context detection and context determination.

\section{3) Distributed approach}

This splits the context detection process, with the subsystems or their configuration modules compiling a series of statistical parameters, such as means and variances. These are then sent to the context determination module, which uses them to detect the context. This approach should enable subsystem suppliers to protect their IP and is efficient in terms of the amount of data conveyed between modules. However, it requires standardization of the statistical parameters used for context detection. At present, context detection is insufficiently mature for the optimum set of descriptors to be known.

Further research is needed to determine the best way forward. It is possible that different approaches to context detection may be needed for different classes of subsystem.

\section{AmBIGUITY}

\section{A. The Problem}

Ambiguity occurs when measurements can be interpreted in more than one way, leading to different navigation solutions, only one of which is correct. Any navigation technique can potentially produce ambiguous measurements. The likelihood depends on both the positioning method and the context, both environmental and behavioral. Urban and indoor positioning techniques that do not require dedicated infrastructure are particularly vulnerable to ambiguity. Poor handling of ambiguity results in erroneous navigation solutions and the navigation system can become 'lost', whereby it is unable to recover and may even reject correct measurements.

There are six main causes of ambiguity: feature identification, pattern matching, propagation anomalies, geometry, system reliability, and context ambiguity. Each of these is described in turn in the following subsections.

\section{1) Feature identification ambiguity}

The proximity, ranging, angular positioning, and Doppler positioning methods all use landmarks for positioning. These may be radio, acoustic, or optical signals, or natural or manmade features of the environment. For reliable positioning, these signals or features must be correctly identified. 
Digital signals intended for positioning incorporate identification codes. However, where a signal is weak and/or interference is high, it may be possible to use the signal for positioning but not decode the identification information. For signals of opportunity, i.e. not designed for positioning, the identification codes may be encrypted, while analog signals do not typically have identifiers. These signals must be identified using their frequencies and an approximate user position, in which case there may be multiple candidates. Even where a signal of opportunity is identifiable, the transmission site may change without warning. For example, Wi-Fi access points are sometimes moved and mobile phone networks are periodically refigured. Thus, there is a risk of false landmark identification.

Environmental features are difficult to identify uniquely. In image-based navigation, man-made features, such as roads, buildings, and signs, are easiest to identify in images due to their line and corner features. However, similar objects are often repeated in relatively close proximity. For example, Fig. 18 shows the locations of the five 'No entry' signs in a $1200 \mathrm{~m}$ circuit of Central London streets [61]. Two of the signs are within $20 \mathrm{~m}$ of each other.

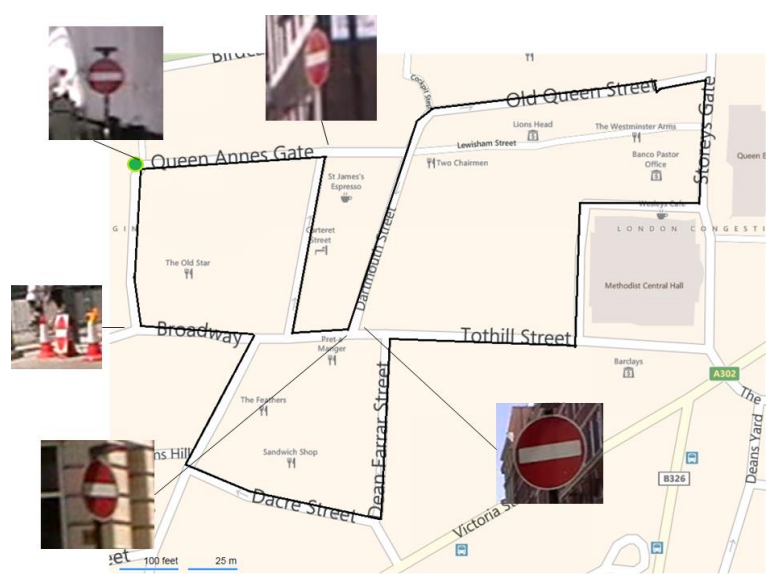

Figure 18. 'No entry' signs in a $1200 \mathrm{~m}$ circuit of Central London (background image courtesy of Bing maps)

\section{2) Pattern-matching ambiguity}

The pattern-matching positioning method maintains a database of measurable parameters that vary with position. Examples include terrain height, magnetic field variations, WiFi signal strengths, and GNSS signal availability information. Values measured at the current unknown user position are compared with predictions from the database over a series of candidate positions. The position solution is then obtained from the highest-scoring candidate(s) [1].

An inherent characteristic of pattern matching is that there is sometimes a good match between measurements and predictions at more than one candidate position. Fig. 19 and Fig. 20 show GNSS shadow-matching scoring maps based on smartphone measurements taken at the same location 40s apart. The scores are obtained by comparing GNSS signal-to-noise measurements with signal availability predictions derived from a 3D city model as described in [101]. In Fig. 19, maximum scores (shown in dark red) are only obtained in the correct street, whereas in Fig. 20, there is also a high-scoring area in the adjacent street, giving two possible position solutions.

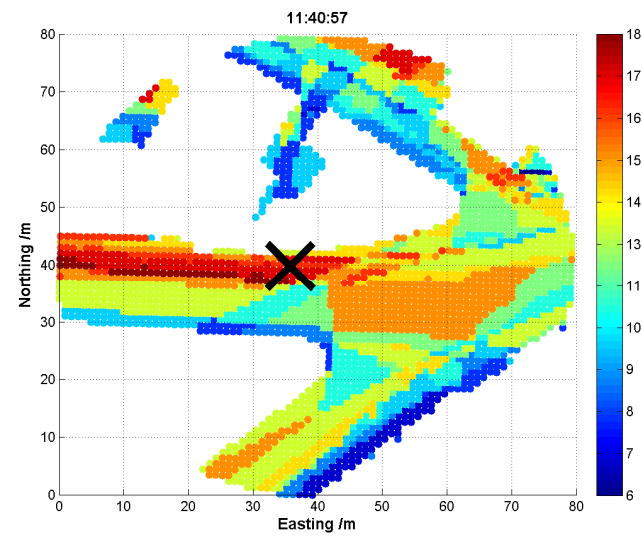

Figure 19. GNSS shadow-matching scoring map - unambiguous case (the cross shows the true position and white areas are indoor locations)

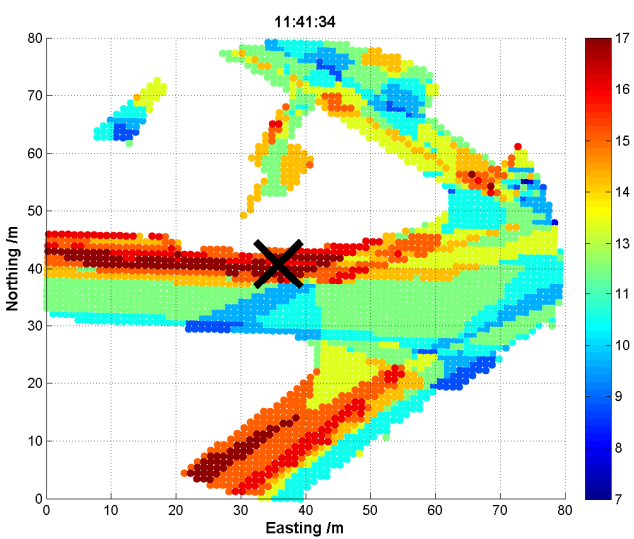

Figure 20. GNSS shadow-matching scoring map - unambiguous case (the cross shows the true position and white areas are indoor locations)

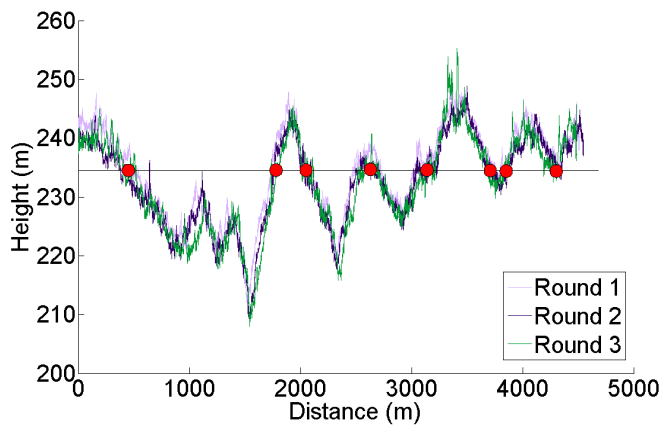

Figure 21. Height of a car derived from a barometric altimeter at three different times; readings of around $235 \mathrm{~m}$ are highlighted.

Fig. 21 presents another example, showing the height of a road vehicle derived from a barometric altimeter at three different times [61]. Provided the altimeter is regularly calibrated, it may be used for terrain-referenced navigation (TRN), determining the car's position along the road by comparing the measured height with a database [1]. However, if only the current height is compared, it will typically match the database at multiple locations within the search area, as the figure shows. The ambiguity can be reduced by comparing a series of measurements from successive epochs, known as a transect, with the database. This approach is applicable to any pattern-matching technique. However, increasing the transect 
length to reduce the ambiguity also reduces the update rate and the ambiguity problem can never be eliminated completely.

\section{3) Signal propagation anomalies}

The ranging, angular positioning, and Doppler positioning methods all make the assumption that the signal propagates from the transmitter (or other landmark) to the user in a straight line at constant speed. Significant position errors can therefore arise when these assumptions are not valid due to phenomena such as non-line-of-sight reception, multipath interference, and severe atmospheric refraction [1]. In challenging environments, such as dense urban areas and indoors, multiple signals are typically affected by propagation anomalies and it is not always easy to determine which signals are contaminated.

Where the position solution is overdetermined (i.e., more than the minimum number of signals are received), different combinations of signals will produce different position solutions when there are significant propagation anomalies. Fig. 22 and Fig. 23 illustrate this for conventional GNSS positioning using a Leica Viva geodetic receiver, showing the position errors obtained using different combinations of GPS and GLONASS signals. In Fig. 22, the receiver is located on a high rooftop [102] and the majority of position solutions are within $15 \mathrm{~m}$ of the mean, with the remainder easily dismissible as outliers. However, in Fig. 23, where the receiver is located in a dense urban location [71], the candidate position solutions are spread over more than $100 \mathrm{~m}$ and the correct position solution is not clear. The densest cluster of positions is far from both the centroid and the truth. Therefore, anomalous signal propagation may be treated as an ambiguity problem.

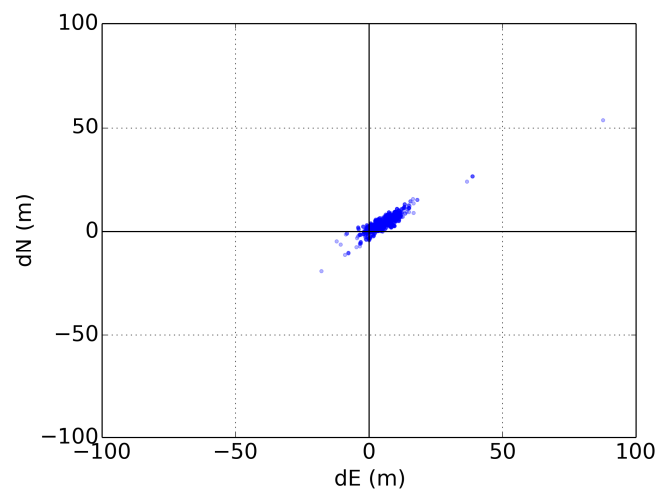

Figure 22. GNSS position errors using different combinations of signals in a rooftop environment

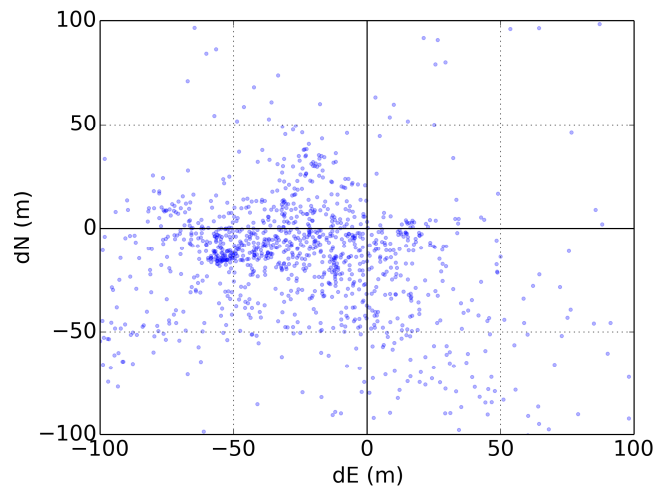

Figure 23. GNSS position errors using different combinations of signals in a dense urban environment

\section{4) Geometric Ambiguity}

Geometric ambiguity occurs when more than one position solution may be derived from a set of otherwise unambiguous measurements. Fig. 24 shows two examples. On the left, two ranging measurements in two dimensions produce circular lines of position that intersect in two places. On the right, a ranging measurement and a direction-finding measurement are made using the same signal. As direction finding has a $180^{\circ}$ ambiguity, the lines of position also intersect at two places.
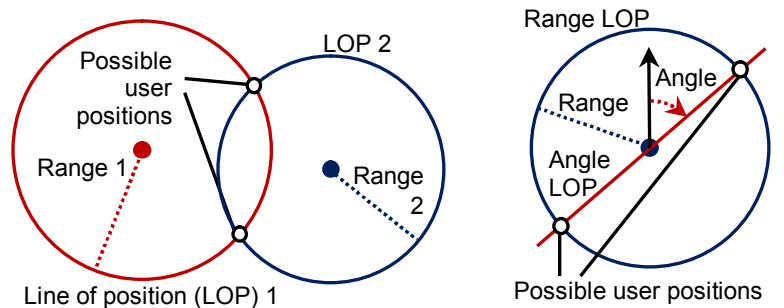

Figure 24. Geometric ambiguity in two dimensions from two ranging measurements (left), and a ranging and direction-finding measurement (right)

\section{5) System Reliability}

Navigation subsystems can produce incorrect information for a host of different reasons. Some examples include:

- User equipment hardware and software faults;

- Transmitter hardware and software faults;

- Out-of-date databases used for pattern matching, including TRN, GNSS shadow matching, and map matching;

- Wheel slips in odometry;

- The effects of passing vehicles and animals on environmental feature visibility, availability and strength of radio signals, and Doppler-based dead reckoning.

Some of these failure modes are easily detectable through the measurements failing basic range checks [1] or being absent altogether. In other cases, faults may be detected by consistency checks [1] within the subsystem. For example, wheel slip may be detected by comparing measurements from different wheels, while Doppler radar and sonar systems typically incorporate a redundant beam to enable the interruption of a beam by a vehicle or animal to be detected.

Subsystems can sometimes output incorrect information that is plausible. An ambiguity thus exists where it is uncertain whether or not a measurement may be trusted. An ambiguity also exists where a fault has been detected, but not its source. Thus, some of the information produced by the subsystem must be incorrect, but some of it may be correct.

\section{6) Context Ambiguity}

As discussed in Section III, the optimum way of processing sensor information depends on the context. However, if context information is used, the navigation solution will then depend on the assumed context. For example, if an indoor environment is assumed, indoor radio positioning and map matching algorithms that are only capable of producing an indoor position solution may be used. Similarly, if an urban environment is assumed, GNSS shadow matching and outdoor map matching may be selected, resulting in an outdoor position 
solution. Adoption of pedestrian and vehicle motion constraints can also lead to different navigation solutions.

Context determination is not a completely reliable process. Therefore, to minimize the impact of incorrect context assumptions on the navigation solution, the context should be treated as ambiguous whenever there is significant uncertainty.

\section{B. Possible Solutions}

There is no obvious solution to the ambiguity problem. Instead different approaches to integrating ambiguous information may be adopted depending on the relative priorities of solution availability, reliability, and processing load [1]. The main approaches, illustrated in Fig. 25, are discussed in the following subsections. They all require the subsystems to present the different measurement hypotheses and their associated probabilities to the integration algorithm.

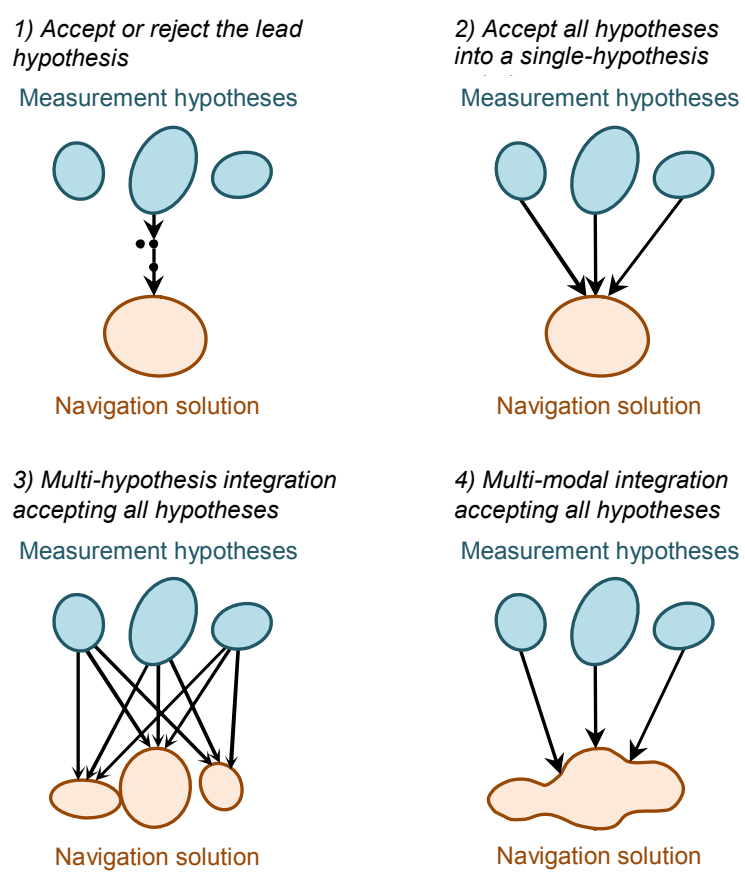

Figure 25. Methods of handling ambiguous measurements in a navigation integration algorithm

\section{1) Accept or reject the lead hypothesis}

The simplest way of handling ambiguous information is to maintain a single-hypothesis navigation solution and consider only the most-probable hypothesis from each subsystem. This is then accepted or rejected based on the following criteria:

- Whether the probability of the highest-scoring hypothesis above a certain threshold.

- Whether the probability of the second-highest-scoring hypothesis below a certain threshold.

- Whether the highest-scoring measurement hypothesis is consistent with the current integrated navigation solution. (Determinable using measurement innovation filtering [1].)

Context may be incorporated into this approach by accepting the highest-scoring behavioral and environmental contexts where they meet the above criteria and computing a context-independent navigation solution otherwise.

This approach is processor-efficient, but high integrity and availability cannot be achieved simultaneously. Low acceptance thresholds provide high reliability by rejecting most erroneous measurements, but low solution availability as many good measurements are also rejected. Conversely, high acceptance thresholds provide availability at the expense of reliability.

2) Accept all hypotheses into a single-hypothesis solution

A probabilistic data association filter (PDAF) [1][103][104] accepts multiple measurement or context hypotheses, weighting them them according to their probabilities, but represents the navigation solution as the mean and covariance of a uni-modal distribution. The measurement update to the state estimation error covariance matrix accounts for the spread in the hypotheses such that the state uncertainties can sometimes increase following a measurement update.

This approach reconciles the demands of integrity and availability at the price of a moderate increase in processing load. However, the uni-modal navigation solution can sometimes be misleading. For example, if a pattern-matching system determines that the user is equally likely to be in one of two parallel streets, the overall position solution will be midway between those streets.

\section{3) Multi-hypothesis integration accepting all hypotheses}

Multi-hypothesis integration deals with multiple measurement and context hypotheses by spawning multiple integration filters, one for each hypothesis [1][105]. Each filter is allocated a probability based not only on the probabilities of the measurements input to it, but also on the consistency of those measurements with the prior estimates of that filter. This consistency-based scoring is essential; otherwise the filter hypothesis that inputs the highest-scoring measurement hypotheses will always dominate, regardless of whether those measurements are consistent across subsystems and successive epochs.

A fundamental characteristic of multi-hypothesis filtering is that the number of hypotheses grows exponentially from epoch to epoch. This is clearly impractical so the number of hypotheses is limited by merging the lowest-scoring hypotheses into higher-scoring neighbors.

The overall navigation solution is the weighted sum of the constituent filter hypotheses. Each individual filter hypothesis describes a uni-modal distribution. However, the combined navigation solution is multi-modal. Thus, the position probability can be higher in two streets than the in the buildings between those streets. This is a clear advantage over the PDAF-based approach, but the processing load is higher.

\section{4) Multi-modal integration accepting all hypotheses}

A multi-modal filter is not constrained to model the states it estimates in terms of a mean and covariance. This enables it to process multiple measurement and/or context hypotheses and represent the result as a weighted sum of the probability distributions arising from the individual hypotheses. Suitable data fusion algorithms include the Gaussian mixture filter and 
the particle filter. A key advantage over multi-hypothesis integration is that measurements may be treated as continuous probability distributions instead of as a set of discrete hypotheses. This enables pattern-matching measurements to be integrated more naturally and offers greater flexibility in handling signal propagation anomalies.

A Gaussian mixture filter models the probability distribution of the navigation solution as the weighted sum of a series of multi-variate Gaussian distributions. An example is the iterative Gaussian mixture approximation of the posterior (IGMAP) technique, which has been applied to terrain referenced navigation integrated with inertial navigation [77].

A particle filter models the probability distribution of the navigation solution using a series of semi-randomly distributed samples, known as particles. Between a thousand and a million particles are typically deployed, with a higher density of particles in higher-probability regions of the distribution [1][106][107][108]. Particle filters have been used with a number of different navigation technologies, including TRN [109], pedestrian map matching [110], Wi-Fi positioning [4], and GNSS shadow matching [111].

Multi-modal integration algorithms offer the greatest flexibility in reconciling the demands of solution availability and reliability, but also potentially impose the highest processing load.

\section{Issues to Resolve}

The key challenge in handling ambiguous measurements is determining realistic probabilities for each hypothesis. A probability must also be calculated for the null hypothesis, i.e. the hypothesis that every candidate measurement output by the subsystem is wrong. The same applies to ambiguous context.

A feature identification algorithm must allocate a score to every database feature that it compares with the sensor measurements In practice, only features within a predefined search area, based on the prior position solution and its uncertainty, will be considered. Features scoring above a certain threshold will be possible matches. Similarly, patternmatching algorithms allocate a score to each candidate position in the search area according to how well the sensor measurements match the database at that point. For correct handling of ambiguous matches, these scores should be as close as possible to the probabilities of the feature match or candidate position being correct.

Feature identification and pattern-matching algorithms can also fail to consider the correct feature or candidate position for several reasons. The correct feature or position may be outside the database search area. It may be absent due to the database being out of date. The sensor may also observe or be affected by a temporary feature that is not in the database, such as a vehicle. The null hypothesis probability must account for all of these possibilities. In practice, it will be higher where there is no good match between the measurements and database.

Signal propagation anomalies affect the error distributions of ranging, angle, and Doppler shift measurements, and the positions and velocities derived from them. These error distributions depend on whether the signals are direct line-of- sight (LOS), non-line-of-sight (NLOS), or multipathcontaminated LOS. However, this is not typically known. Signal strength measurements, environmental context, signal elevation (for GNSS), distance from the transmitter (for terrestrial signals), consistency between different measurements, and 3D city models can all contribute useful information. However, their relationship with the measurement errors is complex, so a semi-empirical approach is needed.

Moving on to reliability, virtually any subsystem can produce false information. The overall probability will typically be very low and thus only significant for highintegrity applications. However, the failure probability will be higher in certain circumstances, in which case the relevant subsystem should report a higher null probability. For example, in odometry, the probability of a wheel slip depends on host vehicle dynamics. Similarly, a radio signal is more likely to be faulty if it is weaker than normal. Repeated measurements, changes to the update interval and sudden changes in a sensor output are also indicative of potential faults.

Geometric ambiguity is easy to quantify as the candidate solutions have equal probability in the absence of additional information.

As proposed in Section III.D.2), the context determination process should produce multiple context hypotheses, each with an associated probability. Therefore, it is important to ensure that all navigation subsystems that use this context information do so in a probabilistic manner. Thus, where different context hypotheses lead to different values of the measurements output by a navigation subsystem, each measurement hypotheses should be accompanied by a probability derived from the context probabilities.

A further issue to resolve is the relationship between discrete and continuous ambiguity. Ambiguities in feature identification, solution geometry, failures, and context categorization are discrete and are suited to integration filters that treat them as a set of discrete hypotheses. However, the position solution ambiguity in pattern-matching is continuous, i.e. the probability density is a continuous function of position, albeit sampled at discrete grid points. This probability distribution may be input directly to a particle filter. However, if the integration algorithm is a uni-modal filter or a bank of uni-modal filters, the probability distribution must be converted to a set of discrete hypotheses. This can be done by fitting a set of Gaussian distributions to the probability distribution. For signal propagation anomalies, their presence or absence is discrete. However, the resulting measurement error distribution is continuous, so a similar approach is appropriate.

\section{Ambiguity and Modular Integration}

The same challenging environments that require multiple navigation subsystems to maximize solution availability, accuracy, and reliability can also induce those subsystems to produce ambiguous measurements. Consequently, the modular integration architecture proposed in Section II.B should be capable of handling ambiguous measurements.

Determination of the hypothesis and null probabilities must be the responsibility of the subsystem suppliers as they have 
the necessary specialist knowledge. The probabilities may be calculated within either the subsystems or in the configuration modules. The integration filter must then be designed to accept multi-hypothesis measurements, handling then using one of the methods described in Section III.B.

In principle, almost any measurement can be ambiguous. Even dead-reckoning systems are subject to reliability issues and context ambiguity. Therefore, the interface standard for communication between the configuration and filter modules (see Section II.C) should support multiple hypotheses of any measurement type. A maximum number of hypotheses per measurement should be agreed.

Where context ambiguity leads to measurement ambiguity, multiple subsystems may be affected such that hypotheses are correlated across those subsystems. To enable the integration algorithm to handle these measurements correctly, the interface standard should support the tagging of measurement hypotheses with context information, where appropriate.

To support the optimal integration of pattern-matching techniques and signal propagation anomalies, the interface standard should also support the representation of position, ranging and angular measurements as probability distributions.

\section{E. Ambiguity and Integrity}

For high-integrity applications, two requirements must be met. The first is that the probability of the position error exceeding a certain limit, known as the alert limit, due to an undetected fault is below a certain very low value. For example, for civil aviation in the nonprecision approach phase, the probability of the horizontal position error exceeding $556 \mathrm{~m}$ during any one-hour period due to any one failure mode must be less than $10^{-7}$. Therefore, in handling ambiguous measurements, hypotheses with very low probabilities must be considered. Similarly, large database search areas are required for feature identification and pattern matching.

The second requirement of high-integrity applications is that when faulty information is detected, a navigation solution that is isolated from the source of the faulty information must be generated and that navigation solution verified as fault-free. This requires an array of parallel navigation filters to be maintained, each excluding one or more signal or subsystem. Parallel filters may also be required for different hypotheses of the same measurement when combining hypotheses could compromise the alert limit.

In practice, the processing capacity needed to incorporate potentially ambiguous measurements in a high-integrity navigation system is likely to be prohibitively expensive. Consequently practical high-integrity navigation in challenging environments may require dedicated positioning infrastructure.

\section{ENVIRONMENTAL DATA}

\section{A. The Problem}

Position-fixing systems need information about the environment, sometimes known as a 'world model' [112], to operate. Proximity, ranging, and angular positioning, all use landmarks that must be identified. For GNSS and other long- range radio systems, identification codes are determined when the system is designed and incorporated in the user equipment. However, this is not practical for shorter range signals, whether opportunistic or designed for positioning, due to the vast numbers of transmitters available worldwide and the fact that many will be installed during the lifetime of the user equipment. The user equipment will also require information on the characteristics of a signal to enable it to use that signal for ranging. A mobile device equipped with a generic radio or transceiver may be required to download software to enable it to use a proprietary indoor positioning system. For environmental feature matching techniques, the user equipment requires information to enable it to identify each landmark.

Navigation using landmarks also requires their positions and, for passive ranging, their timing offsets. Signals designed for positioning typically provide this information, but it can take a long time to download (30s for GPS C/A code) and can be difficult to demodulate under poor reception conditions. The positions of opportunistic radio transmitters and environmental features must be determined by other means.

For positioning using the pattern-matching method, a measurement of radio signal strength or a characteristic of the environment, such as the terrain height or magnetic field, is compared with a database to determine position [3][4][33][34] [61][77][78]. Therefore, a database providing values of the measured parameter over a regular grid of positions is required. Map matching requires a map database to indicate where the user can and cannot go [26][27][81]. GNSS shadow matching requires a $3 \mathrm{D}$ city model to predict signal visibility [38].

Finally, as discussed in Section II, mapping is required to determine environmental context information from the position solution and to enable location-dependent context connectivity information (e.g., the location of train stations) to be used for context determination.

\section{B. Possible Solutions}

The environmental data collection and its distribution to the user equipment are discussed in turn.

\section{1) Data Collection}

Positioning data may be collected either from a systematic survey or by the users. In either case, regular updates will be required. A systematic survey might be conducted by the subsystem supplier, a national mapping agency, or a private third party. The user will need to pay for the data in some way. It could be included in the equipment cost, via a subscription payment, by accepting advertising, or through general taxation (for some national mapping agency data). For mobile devices, such as smartphones, mapping data may be available for some applications, but not others.

Single-user data collection does not involve user charges, but only provides data for places the user has already visited. A simple approach requires a good position solution to collect mapping data. This can work for applications which normally use GNSS, but require backups for temporary outages [61]. However, it does not work for areas where GNSS reception is poor. Simultaneous localization and mapping (SLAM) techniques can perform mapping without a continuous position 
solution. However, there are several constraints. Firstly, a good position solution that is independent of the data being mapped is required at some point, usually the start. Secondly, a navigation system including dead-reckoning must be used technology. Thirdly, locations must be visited repeatedly within a short period of time (to achieve 'loop closure'). Finally, only features close to the user can be mapped.

Cooperative mapping by a group of users solves many of the problems of single-user mapping [113]. It can provide individual users with data for places they have not visited before. Distant landmarks can also be mapped more easily by multiple users, particularly where it is necessary to determine a timing offset as well as the location. However, a method for comparing and combining data from multiple users is required.

\section{2) Data Distribution}

For data collected by a systematic survey, there are two main data distribution models: pre-loading and streaming. Preloading requires sufficient user equipment data storage to cover the area of operation. New data may have to be loaded prior to a change in operating area and updates will be required. However, a continuous communications link is not needed.

Streaming requires much less data to be stored by the user and provides up-to-date information, but only where a communications link is available. Although buffering can bridge short outages, navigation data is simply not available for areas without sufficient communications coverage. Continuous streaming can also be expensive. One solution is a cooperative approach using peer-to-peer communications for much of the data distribution. A pair of users traveling in opposite directions along the same route will each have data that is useful to the other. A further possibility is to incorporate local information servers in Wi-Fi access points for exchanging information relevant to the immediate locality. This might be best suited to indoor navigation, where there is an incentive for the building operator to provide the service.

For data collected by a single user, no data distribution is required other than a back-up. For cooperative data collection by multiple users, a method of data exchange is needed. This can be via a central server, communicating either in real time or whenever the user returns to base. It can also be through peer-to-peer communications or through local information servers, where there is an incentive to provide them.

\section{Issues to Resolve}

Standardization is a major part of the data management challenge. A multisensor navigation system will typically incorporate multiple subsystems with data requirements. This might include road or building mapping, radio signal information, terrain height, magnetic anomalies, visual landmarks, and building signal-masking information for GNSS shadow matching. There will be a different standard for each type of data. Furthermore, different subsystem suppliers will often use different standards for the same type of data. This is sometimes done for commercial and/or security reasons, so the data may be encrypted. There may also be technical reasons for different data standards. For example, in image-based navigation, different feature recognition algorithms require different descriptive data.
Ideally, all navigation data in a multisensor system should be distributed by the same method. This requires agreement of storage and communication protocols that can handle many different data formats, including encrypted proprietary data and future data formats. Open standards for each type of data should also be agreed, noting that consumer cooperative positioning using peer-to-peer communications and/or local information servers is probably only practical with open data formats. Ideally, the standards should be scalable to enable precisions, spatial resolutions, and search areas to be adapted to the available data storage and communications capacity.

Peer-to-peer data exchange requires a suitable communications link. Bluetooth is the established standard for consumer applications. Classic Bluetooth provides sufficient capacity, but it takes longer to establish a connection than passing pedestrians or vehicles remain within range. Bluetooth low energy can establish a connection quickly, but the data capacity is limited to $100 \mathrm{kbit} / \mathrm{s}$. This is sufficient for some kinds of navigation data, but not others. Professional and military users have more flexibility to select suitable datalinks.

Finally, establishing local information servers requires both standardization and an incentive for the hosts. Demand would be greater if there were applications beyond navigation and positioning. Possibilities include product information in shops and exhibit information in museums, both of which might be provided more efficiently from a local server than the internet. For home users to provide local information servers, they would also have to benefit from them, a potential "chicken and egg" problem. For military applications, local information servers are a potential security risk and a target for attack.

\section{CONCLUSIONS AND RECOMMENDATIONS}

Achieving accurate and reliable navigation in challenging environments without additional infrastructure requires complex multisensor integrated navigation systems. However, implementing them presents four key challenges: complexity, context, ambiguity, and environmental data handling. Each of these problems has been explored and solutions proposed. Here, the main conclusions and recommendations for standardization and further research are summarized.

\section{A. Conclusions}

A modular integration architecture, comprising a universal integration filter module and a configuration module for each subsystem enables multiple subsystems to be integrated without the need for whole-system expertise in a single organization. It also enables subsystems from different organizations to be combined without sharing intellectual property and allows new navigation technologies and methods to be added without having to redesign the whole system.

Context-adaptive navigation enables a navigation system to respond to changes in the environment and host-vehicle (or user) behavior, deploying the most appropriate algorithms for the current circumstances. Context can be determined more reliably by adopting a probabilistic approach and using connectivity, association, and scope information. The potential use of GNSS to distinguish between indoor and outdoor environments and Wi-Fi to detect indoor-outdoor transitions 
has been demonstrated. The potential use of accelerometer vibration spectra to distinguish between a car and a train and between an elevator and an escalator has also been shown.

Navigation solution ambiguity can arise from feature identification, pattern matching, propagation anomalies, solution geometry, system reliability issues, and context ambiguity. To handle ambiguity in a multisensor navigation system, the subsystems must present the different measurement hypotheses and their associated probabilities to the integration algorithm. The best strategy for integrating ambiguous measurements depends on the relative priorities of solution availability, reliability, and processing load. Options include simply accepting or rejecting the lead hypothesis, accepting all measurement hypotheses into a single-hypothesis solution, multi-hypothesis integration accepting all hypotheses, and multi-modal integration accepting all hypotheses. For veryhigh-integrity applications, the processing capacity needed to incorporate potentially ambiguous measurements is likely to be prohibitively expensive.

Position-fixing subsystems need data such as locations of radio transmitters and other landmarks, information for identifying signals and landmarks, road or building mapping, terrain height, magnetic anomalies, and building signalmasking information (for GNSS shadow matching). Different models for collecting and distributing this data suit different applications. Data may be collected from a systematic survey or by users. In the latter case, users may cooperate to share data where a suitable communication link is available. Data may be stored by users in advance, streamed from central servers, and/or distributed cooperatively using peer-to-peer communication and/or local information servers.

\section{B. Standardization}

To enable effective communication between modules from different suppliers, an open-standard interface specification should be developed to convey the following information:

- The integrated navigation solution;

- Measurements from navigation subsystems, including error specifications, multiple hypotheses with their associated probabilities, and the option to represent position, ranging and angular measurements as probability distributions;

- Feedback of subsystem error calibration information;

- Accuracy, integrity, continuity, solution availability, update rate, and power consumption requirements;

- Integration filter capability specifications;

- Environmental and behavioral context hypotheses and their associated probabilities;

- Context detection information from the subsystems;

- Data for use by the subsystems, including road or building mapping, radio signal information, terrain height, magnetic anomalies, and visual landmarks.

Standards are also needed for peer-to-peer communication of navigation data and Wi-Fi local information servers.

\section{Further Research}

Further research is needed to support the standardization process described above, including the identification of a set of fundamental measurement types and their error sources, and the establishment of the best set of context categories for integrated navigation.

Extensive research into context detection and determination is needed, including the measurements to use, the statistical parameters to derive from those measurements, and a set of context association and connectivity rules.

An assessment of the different methods for handling ambiguous measurements is needed, comparing accuracy, reliability, solution availability, and processing load. This will enable the community to determine which methods are suited to different applications.

Finally, there is a need for a practical demonstration of the key concepts proposed in this paper, including modular integration, context adaptivity, ambiguous measurement handling, and collection and distribution of environmental data.

\section{REFERENCES}

[1] Groves, P. D., Principles of GNSS, Inertial and Multisensor Integrated Navigation Systems, $2^{\text {nd }}$ Ed., Artech House, 2013.

[2] Farrell, J. A., Aided Navigation: GPS with High Rate Sensors, McGraw Hill, 2008.

[3] Hatami, A., and K. Pahlavan, "A Comparative Performance Evaluation of RSS-Based Positioning Algorithms Used in WLAN Networks," IEEE Wireless Communications and Networking Conference, March 2005.

[4] Faragher, R. M., C. Sarno, and M. Newman, "Opportunistic Radio SLAM for Indoor Navigation using Smartphone Sensors," Proc. IEEE/ION PLANS, Myrtle Beach, SC, April 2012.

[5] Nur, K., et al., "Application of the Improved FOCUSS for Arrival Time Estimation (IFATE) Algorithm to WLAN High Accuracy Positioning Services," Proc. 2nd International Conference and Exhibition on Ubiquitous Positioning, Indoor Navigation and Location-Based Service (UPINLBS), Helsinki, Finland, October 2012.

[6] Sahinoglu, Z., S. Gezici, and I. Guvenc., Ultra-wideband Positioning Systems: Theoretical Limits, Ranging Algorithms, and Protocols, Cambridge University Press, 2008.

[7] Bensky, A., Wireless Positioning Technologies and Applications, Artech House, 2008.

[8] Bhattacharrya, T., et al., "Location by Database: Radio-Frequency Pattern Matching," GPS World, June 2012, pp. 8-12.

[9] Duffett-Smith, P. J., and P. Hansen, "Precise Time Transfer in a Mobile Radio Terminal,” Proc. ION NTM, San Diego, CA, January 2005.

[10] Rabinowitz, M., and J. J. Spilker, Jr., “A New Positioning System Using Television Synchronization Signals," IEEE Transactions on Broadcasting, Vol. 51, 2005, pp. 51-61.

[11] Palmer, D., et al., "Radio Positioning using the Digital Audio Broadcasting (DAB) Signal,” Journal of Navigation, Vol. 64, 2011, pp. 45-59.

[12] Thevenon, P., et al., "Positioning Using Mobile TV Based on the DVBSH Standard," NAVIGATION, Vol. 58, 2011, pp. 71-90.

[13] Mathews, M. B., P. F. Macdoran, and K. L. Gold, "SCP Enabled Navigation Using Signals of Opportunity in GPS Obstructed Environments," NAVIGATION, Vol. 58, 2011, pp. 91-110.

[14] Webb, T. A., P. D. Groves, R. J. Mason, and J H. Harrison, “A New Differential Positioning Technique Applicable to Generic FDMA Signals of Opportunity," Proc. ION GNSS 2011, Portland OR. Also available from http://discovery.ucl.ac.uk/.

[15] Kalliola, K., "High Accuracy Indoor Positioning Based on BLE," Nokia Research Center Presentation, April 27, 2011. 
[16] Hirokawa, R., et al., "Autonomous Vehicle Navigation with Carrier Phase DGPS and Laser-Scanner Augmentation," Proc. ION GNSS 2004, Long Beach, CA.

[17] Campbell, J. L., M. Uijt de Haag, and F. van Graas, "Terrain Referenced Positioning using Airborne Laser Scanner," NAVIGATION, Vol. 52, 2005, pp. 189-197.

[18] Campbell, J. L., et al., "Flash-LADAR Inertial Navigator Aiding," Proc. IEEE/ION PLANS, San Diego, CA, April 2006, pp. 677-683.

[19] Neregård, F., et al., "Saab TERNAV, an Algorithm for Real Time Terrain Navigation and Results from Flight Trials using a Laser Altimeter," Proc. ION GNSS 2006, Fort Worth, TX.

[20] Joerger, M., and B. Pervan, "Range-Domain Integration of GPS and Laser-scanner Measurements for Outdoor Navigation," Proc. ION GNSS 2006, Fort Worth, TX.

[21] Soloviev, A., D. Bates, and F. van Graas, "Tight Coupling of Laser Scanner and Inertial Measurements for a Fully Autonomous Relative Navigation Solution," NAVIGATION, Vol. 54, 2007, pp. 189-205.

[22] Judd, T., "A Personal Dead Reckoning Module," Proc. ION GPS-97, Kansas, MO, 1997.

[23] Ladetto, Q., "On Foot navigation: continuous step calibration using both complementary recursive prediction and adaptive Kalman filtering," Proc. ION GPS 2000, Salt Lake City, UT.

[24] Leppäkoski, H., et al., "Error Analysis of Step Length Estimation in Pedestrian Dead Reckoning," Proc. ION GPS 2002, Portland, OR September 2002, pp. 1136-1142.

[25] Mather, C. J., P. D. Groves, and M. R. Carter, "A Man Motion Navigation System Using High Sensitivity GPS, MEMS IMU and Auxiliary Sensors," Proc. ION GNSS 2006, Fort Worth, TX.

[26] Woodman, O., and R. Harle, "Pedestrian Localisation for Indoor Environments," Proc. UbiComp'08, Seoul, Korea, September 2008.

[27] Beauregard, S., Widyawan, and M. Klepal, "Indoor PDR Performance Enhancement using Minimal Map Information and Particle Filters," Proc. IEEE/ION PLANS, Monterey, CA, May 2008.

[28] Blanchart, P., L. He, and F. Le Gland, "Information Fusion for Indoor Localization," Proc $12^{\text {th }}$ International Conference on Information Fusion, Seattle, WA, July 2009.

[29] Abdulrahim, K., et al., "Aiding Low Cost Inertial Navigation with Building Heading for Pedestrian Navigation," Journal of Navigation, Vol. 64, pp. 219-233.

[30] Aggarwal, P., et al., "Map Matching and Heuristic Elimination of Gyro Drift for Personal Navigation Systems in GPS-denied Conditions," Measurement Science and Technology, Vol. 22, 2011, paper 025205.

[31] Robertson, P., M. Garcia Puyol, and M. Angermann, "Collaborative Pedestrian Mapping of Buildings Using Inertial Sensors and FootSLAM," Proc. ION GNSS 2011, Portland, OR.

[32] Judd, T., and T. Vu, "Use of a New Pedometric Dead Reckoning Module in GPS Denied Environments," Proc. IEEE/ION PLANS, Monterey, CA, May 2008.

[33] Gozick, B., et al., "Magnetic Maps for Indoor Navigation,” IEEE Trans. on Instrumentation and Measurement, Vol. 60, 2011, pp. 3883-3891.

[34] Shockley, J. A., and J. F., Raquet, "Three-Axis Magnetometer Navigation in Suburban Areas," Proc. ION GNSS 2012, Nashville, TN.

[35] Gusenbauer, D., C. Isert, and J. Krösche, "Self-Contained Indoor Positioning on Off-The-Shelf Mobile Devices," Proc. Indoor Positioning and Indoor Navigation, Zürich, Switzerland, Sept. 2010.

[36] Khalifa, S., and M. Hassan, "Evaluating Mismatch Probability of Activity-based Map Matching in Indoor Positioning," Proc. Indoor Positioning and Indoor Navigation, Sydney, Australia, November 2012.

[37] Groves, P. D., "Shadow Matching: A New GNSS Positioning Technique for Urban Canyons," Journal of Navigation, Vol. 64, 2011, pp. 95-105. Also available from http://discovery.ucl.ac.uk/.

[38] Wang, L., P. D. Groves, and M. K. Ziebart, "GNSS Shadow Matching: Improving Urban Positioning Accuracy using a 3D City Model with Optimized Visibility Scoring Scheme," NAVIGATION, Vol. 60, 2013 pp. 195-207. Also available from http://discovery.ucl.ac.uk/.

[39] Wang, L., P. D. Groves, and M. K. Ziebart, "Urban Positioning on a Smartphone: Real-time Shadow Matching Using GNSS and 3D City
Models," Inside GNSS, November/December 2013, pp. 44-56. Also available from http://discovery.ucl.ac.uk/.

[40] Suzuki, T., and N. Kubo, "GNSS Positioning with Multipath Simulation using 3D Surface Model in Urban Canyon," Proc. ION GNSS 2012, Nashville, TN.

[41] Yozevitch, R., B. Ben-Moshe, and A. Dvir, "GNSS Accuracy Improvement Using Rapid Shadow Transitions," IEEE Trans. On Intelligent Transportation Systems, DOI: 10.1109/TITS.2013.2294537, 2014

[42] Handley, R. J., L. Dack, and P. McNeil, "Flight Trials of the Continuous Visual Navigation System," Proc. ION NTM, Long Beach, CA, 2001.

[43] Allen, J. W., and D. M. Bevly, "Relating Local Vision Measurements to Global Navigation Satellite Systems Using Waypoint Based Maps," Proc. IEEE/ION PLANS, Palm Springs, CA, May 2010.

[44] Hide, C., T. Botterill, and M. Andreotti, "An Integrated IMU, GNSS and Image Recognition Sensor for Pedestrian Navigation," Proc. ION GNSS 2009, Savannah, GA.

[45] Kessler, C., et al., "Vision-Based Attitude Estimation for Indoor Navigation using Vanishing Points and Lines," Proc. IEEE/ION PLANS, Palm Springs, CA, May 2010.

[46] Veth, M. J., "Navigation Using Images: A Survey of Techniques," NAVIGATION, Vol. 58, 2011, pp. 127-139.

[47] Hide, C., T. Botterill, and M. Andreotti, "Vision-aided IMU for Handheld Pedestrian Navigation," Proc. ION GNSS 2010, Portland, OR.

[48] Kempe, V., Inertial MEMS Principles and Practices, Cambridge University Press, 2011.

[49] Stockton, J. K., K. Takase, and M. A. Kasevich, "Absolute Geodetic Rotation Measurement Using Atom Interferometry," Physical Review Letters, Vol. 107, 2011, Article ID 133001.

[50] McGuinness, H. J., A. K. Rakholia, and G. W. Biedermann, "High DataRate Atom Interferometer for Measuring Acceleration," Applied Physics Letters, Vol. 100, 2012, Article ID 011106.

[51] Donely, E. A., "Nuclear Magnetic Resonance Gyroscopes," Proc. IEEE Sensors 2010, Waikoloa, HI, November 2010, pp. 17-22.

[52] Lo, S. C., and P. K. Enge, "Signal Structure Study for a Passive Ranging System using Existing Distance Measuring Equipment (DME)," Proc. ION ITM, Newport Beach, CA, January 2012.

[53] Li, K., and W. Pelgrum, "Enhanced DME Carrier Phase: Concepts, Implementation, and Flight-test Results," NAVIGATION, Vol. 60, 2013, pp. 209-220.

[54] Hargreaves, C., P. Williams, and M. Bransby, "ASF Quality Assurance for eLoran,” Proc. IEEE/ION PLANS, Myrtle Beach, SC, April 2012.

[55] Enge, P., et al., "Orbital Diversity in Satellite Navigation," Proc. ION GNSS 2012, Nashville, TN.

[56] Van Diggelen, F., A-GPS: Assisted GPS, GNSS, and SBAS, Artech House, 2009.

[57] Barnes, J., et al., "High accuracy positioning using Locata's next generation technology," Proc. ION GNSS 2005, Long Beach, CA.

[58] Manandhur, D., et al., "Development of Ultimate Seamless Positioning System based on QZSS IMES," Proc. ION GNSS 2008, Savannah, GA.

[59] Meiyappan, S., A. Raghupathy, and G. Pattabiraman, "Positioning in GPS Challenged Locations - The NextNav Terrestrial Positioning Constellation," Proc. ION GNSS+ 2013, Nashville TN.

[60] Groves P. D., "The PNT Boom: Future Trends in Integrated Navigation," Inside GNSS March/April 2013 pp. 44-49. Also available from http://discovery.ucl.ac.uk/.

[61] Walter, D. J., Groves, P. D., Mason, R. J., Harrison, J., Woodward, J. and Wright, P., "Novel Environmental Features for Robust Multisensor Navigation," Proc. ION GNSS+ 2013, 2013 Nashiville, TN. Also available from http://discovery.ucl.ac.uk/.

[62] Groves, P. D., "The Complexity Problem in Future Multisensor Navigation and Positioning Systems: A Modular Solution," Journal of Navigation, Vol. 67, 2014, pp. 311-206. Also available from http://discovery.ucl.ac.uk/.

[63] Hide, C., T. Moore, and C. Hill, "A Multi-Sensor Navigation Filter for High Accuracy Positioning in all Environments," Journal of Navigation, Vol. 60, 2007, pp. 409-425. 
[64] Penn, T. R., All Source Sensor Integration Using an Extended Kalman Filter, Air Force Institute of Technology, 2012.

[65] Soloviev, A. and C. Yang, "Reconfigurable Integration Filter Engine for Plug-and-Play Navigation," Proc. ION GNSS+ 2013, Nashville, TN.

[66] Mohammed, A. H., and K. P. Schwarz, "Adaptive Kalman Filtering for INS/GPS,” Journal of Geodesy, Vol. 73, 1999, pp. 193-203.

[67] Wang, J., M. Stewart, and M. Tsakiri, "Online Stochastic Modelling for INS/GPS Integration,” Proc. ION GPS ‘99, Nashville, TN, 1999.

[68] Hide, C., T. Moore, and M. Smith, "Adaptive Kalman Filtering for Low Cost INS/GPS,” Journal of Navigation, Vol. 56, 2003, pp. 143-152.

[69] Berman, Z., Private communication, December 2013.

[70] Shivaramaiah, N. C., and A. G. Dempster, "Cognitive GNSS Receiver Design: Concept and Challenges," Proc. ION GNSS 2011, Portland, OR.

[71] Groves, P. D. and Z. Jiang, "Height Aiding, $C / N_{0}$ Weighting and Consistency Checking for GNSS NLOS and Multipath Mitigation in Urban Areas" Journal of Navigation, Vol. 66, 2013, pp. 653-669. Also available from http://discovery.ucl.ac.uk/.

[72] Obst, M., S. Bauer, and G. Wanielik, "Urban Multipath Detection and mitigation with Dynamic 3D Maps for Reliable Land Vehicle Localization," Proc. IEEE/ION PLANS, Myrtle Beach, SC, April 2012.

[73] Groves, P. D., Z. Jiang, L. Wang, and M. K. Ziebart, "Intelligent Urban Positioning using Multi-Constellation GNSS with 3D Mapping and NLOS Signal Detection," Proc. ION GNSS 2012, Nashville, TN. Also available from http://discovery.ucl.ac.uk/.

[74] Bourdeau, A., M. Sahmoudi, and J.-Y. Tourneret, "Tight Integration of GNSS and a 3D City Model for Robust Positioning in Urban Canyons," Proc. ION GNSS 2012, Nashville, TN.

[75] Peyraud, S., et al., "About Non-Line-Of-Sight Satellite Detection and Exclusion in a 3D Map-Aided Localization Algorithm," Sensors, Vol. 13, 2013, pp. 829-847.

[76] Suzuki, T., and N. Kubo, "Correcting GNSS Multipath Errors Using a 3D Surface Model and Particle Filter," Proc. ION GNSS+ 2013, Nashville, TN.

[77] Runnalls, A. R., P. D. Groves, and R. J. Handley, "Terrain-Referenced Navigation Using the IGMAP Data Fusion Algorithm," Proc. ION $61^{s t}$ Annual Meeting, Boston, MA, June 2005.

[78] Cowie, M., N. Wilkinson, and R. Powlesland, "Latest Development of the TERPROM ${ }^{\circledR}$ Digital Terrain System (DTS)," Proc. IEEE/ION PLANS, Monterey, CA, May 2008.

[79] Nygren, I., "Robust and Efficient Terrain Navigation of Underwater Vehicles," Proc. IEEE/ION PLANS, Monterey, CA, May 2008.

[80] Soehren, W., and W., Hawkinson, "A Prototype Personal Navigation System," Proc. IEEE/ION PLANS, San Diego, CA, April 2006.

[81] Quddus, M. A., W. Y. Ochieng, and R. B. Noland, "Current MapMatching Algorithms for Transport Applications: State-of-the-art and Future Research Directions," Transportation Research Part C, Vol. 15, 2007, pp. 312-328.

[82] Zheng, Y., and P. Cross, "Integrated GNSS with Different Accuracy of Track Database for Safety-critical Railway Control Systems," GPS Solutions, Vol. 16, 2012, pp. 169-179.

[83] Dissanayake, G., et al., "The Aiding of a Low-Cost Strapdown Inertial Measurement Unit Using Vehicle Model Constraints for Land Vehicle Applications," IEEE Trans. on Robotics and Automation, Vol. 17, 2001, pp. 731-747.

[84] Niu, X., S. Nassar, and. N., El-Sheimy, "An Accurate Land-Vehicle MEMS IMU/GPS Navigation System using 3D Auxiliary Velocity Updates," NAVIGATION, Vol. 54, 2007, pp. 177-188.

[85] Lin, T., C. O’Driscoll, and G. Lachapelle, "Development of a ContextAware Vector-Based High-Sensitivity GNSS Software Receiver," Proc. ION ITM, Jan. 2011.

[86] Bancroft, J. B., et al., "Observability and Availability for Various Antenna Locations on the Human Body," Proc. ION GNSS 2010.

[87] Groves, P.D., H. Martin, K. Voutsis, D. Walter, and L. Wang, "Context Detection, Categorization and Connectivity for Advanced Adaptive Integrated Navigation," Proc. ION GNSS+ 2013, Nashville, TN. Also available from http://discovery.ucl.ac.uk/.
[88] Park, C. G., et al., "Adaptive Step Length Estimation with Awareness of Sensor Equipped Location for PNS," Proc. ION GNSS 2007.

[89] Kantola, J., et al., "Context Awareness for GPS-Enabled Phones," Proc. ION ITM, Jan. 2010.

[90] Pei, L., et al., "Using Motion-Awareness for the 3D Indoor Personal Navigation on a Smartphone," Proc. ION GNSS 2011, Portland, OR.

[91] Saeedi, S., et al., "Context Aware Mobile Personal Navigation Using Multi-level Sensor Fusion," Proc. ION GNSS 2011, Portland, OR.

[92] Frank, K., et al., "Reliable Real-Time Recognition of Motion Related Human Activities Using MEMS Inertial Sensors," Proc. ION GNSS 2010, Portland, OR.

[93] Guinness, R. E., "Beyond Where to How: A Machine Learning Approach for Sensing Mobility Contexts Using Smartphone Sensors," Proc. ION GNSS+ 2013, Nashville, TN.

[94] Aggarwal, P., et al., MEMS-Based Integrated Navigation, Artech House, 2010.

[95] Ramanandan, A., et al., "Detection of Stationarity in an Inertial Navigation System," Proc. ION GNSS 2010, Portland, OR.

[96] Marti, E. D., et al., "Context-Aided Sensor Fusion for Enhanced Urban Navigation,” Sensors, Vol. 12, 2012, pp. 16802-16837.

[97] Lin, T., C. O’Driscoll, and G. Lachapelle, "Channel Context Detection and Signal Quality Monitoring for Vector-based Tracking Loops," Proc. ION GNSS 2010, Portland, OR.

[98] Shafiee, M., K., O’Keefe, and G. Lachapelle, "Context-aware Adaptive Extended Kalman Filtering Using Wi-Fi Signals for GPS Navigation," Proc. ION GNSS 2011, Portland, OR.

[99] Stenneth, L., et al., "Transportation mode detection using mobile phones and GIS information," Proc. $19^{\text {th }}$ ACM SIGSPATIAL, November 2011, Chicago, IL.

[100]Quddus, M. A., High Integrity Map Matching Algorithms for Advanced Transport Telematics Applications, $\mathrm{PhD}$ Thesis, Imperial College London, 2006.

[101]Wang, L., P. D. Groves., and M. K. Ziebart, "Smartphone Shadow Matching for Better Cross-street GNSS Positioning in Urban Environments," In preparation for journal submission, 2014.

[102]Jiang, Z., P. Groves, W. Y. Ochieng, S. Feng, C. D. Milner, and P. G. Mattos, "Multi-Constellation GNSS Multipath Mitigation Using Consistency Checking," Proc. ION GNSS 2011, Portland, OR.. Also available from http://discovery.ucl.ac.uk/.

[103]Bar-Shalom, Y., and T. E. Fortmann, Tracking and Data Association, Academic Press, 1988.

[104]Dezert, J., and Y. Bar-Shalom, "Joint Probabilistic Data Association for Autonomous Navigation," IEEE Trans. on Aerospace and Electronic Systems, Vol. 29, 1993, pp. 1275-1285.

[105]Reid, D. B., “An Algorithm for Tracking Multiple Targets," IEEE Trans. on Automatic Control, Vol. AC-24, 1979, pp. 843-854.

[106]Gordon, N. J., D. J. Salmond, and A. F. M. Smith, “A Novel Approach to Nonlinear/Non-Gaussian Bayesian State Estimation," Proc. IEE Radar Signal Process., Vol. 140, 1993, pp. 107-113.

[107]Gustafsson, F. et al., "Particle Filters for Positioning, Navigation and Tracking," IEEE Trans. on Signal Processing, Vol. 50, 2002, pp. 425437.

[108]Ristic, B., S. Arulampalam, and N. Gordon, Beyond the Kalman Filter: Particle Filters for Tracking Applications, Artech House, 2004.

[109]Nordlund, P.-J., and F. Gustafsson, "Marginalized Particle Filter for Accurate and Reliable Terrain-Aided Navigation," IEEE Trans. on Aerospace and Electronic Systems, Vol. 45, 2009, pp. 1385-1399.

[110]Woodman, O., and R. Harle, "Pedestrian Localisation for Indoor Environments," Proc. UbiComp'08, Seoul, Korea, September 2008.

[111]Wang, L., "Kinematic GNSS Shadow Matching Using a Particle Filter," Accepted by ION GNSS+ 2014, Tampa, FL.

[112]Raquet, J. F., "What's Next for Practical Ubiquitous Navigation: World Models and Magnetic Field Maps," Inside GNSS Sept/Oct 2013.

[113]Kasser, Z. M., and T. E. Humphreys, "Observability and Estimability of Collaborative Opportunistic Navigation with Pseudorange Measurements," Proc. ION GNSS 2012, Nashville, TN. 